\title{
RIGIDITY OF ERGODIC VOLUME-PRESERVING ACTIONS OF SEMISIMPLE GROUPS OF HIGHER RANK ON COMPACT MANIFOLDS
}

\author{
GUILLAUME SEYDOUX
}

\begin{abstract}
Let $M$ be a compact manifold, $H$ a semisimple Lie group of higher rank (e.g., $H=S L(n, \mathbf{R})$ with $n \geq 3)$ and $a \in \mathscr{A}(H, M)$ an ergodic $H$-action on $M$ which preserves a volume $v$. Such an $H$-action is conjectured to be "locally rigid": if $a^{\prime}$ is a sufficiently $C^{\prime}$-small perturbation of $a$, then there should exist a diffeomorphism $\Phi$ of the manifold $M$ which conjugates $a^{\prime}$ to $a$. This conjecture would imply that if $\omega$ is an a-invariant geometrical structure on $M$, then there should exist an $a^{\prime}$-invariant geometrical structure $\omega^{\prime}$ on $M$ of the same type. Using Kazhdan property, superrigidity for cocycles, and Sobolev spaces techniques we prove, under suitable conditions, two such results with $\omega=v$ and with $\omega$ a Riemannian metric along the leaves of a foliation of $M$.
\end{abstract}

\section{INTRODUCTION}

Let $M$ be a compact connected manifold and $H$ a Lie group acting on $M$. Unless otherwise specified we always assume that the manifold $M$, geometric structures, and $H$-actions on $M$ we consider are smooth. Let $\mathscr{A}(H, M)$ denote the set of $H$-actions on $M$, endowed for each integer $0 \leq k \leq \infty$ with the $C^{k}$-topology of uniform convergence of $k$-jets on compact subsets of $H$. In general, if $a \in \mathscr{A}(H, M)$, an arbitrary $C^{\infty}$-neighbourhood of $a$, however small, contains actions which are not conjugate to $a:$ take $H=\mathbf{R}, M=T^{2}$, and let $a_{\alpha} \in \mathscr{A}\left(\mathbf{R}, T^{2}\right)$ denote the usual $\mathbf{R}$-action on $T^{2}$ by translations with slope $\alpha \in \mathbf{R}$. Then if $\left(\alpha_{n}\right)_{n \in \mathrm{N}}$ is a sequence of rationals such that $\alpha_{n} \underset{n \rightarrow \infty}{\longrightarrow} \alpha$, $\alpha \notin \mathbb{Q}$, then although $a_{\alpha_{n}} \frac{C^{\infty}}{n \rightarrow \infty} a_{\alpha}$, the $a_{\alpha_{n}}$ are never conjugate to $a_{\alpha}$ (as, for instance, the $a_{\alpha_{n}}$ are not ergodic while $a_{\alpha}$ is). However we have the following conjecture:

Main Conjecture 1.1 (Local rigidity of ergodic volume-preserving actions of semisimple Lie groups of higher rank and their lattice subgroups on compact manifolds). Let $H$ be a connected semisimple Lie group with finite center and such that the rank of each simple factor is at least two. Let $\Gamma \subset H$ be a lattice subgroup (i.e., a discrete subgroup such that the $H$-space $H / \Gamma$ admits a finite invariant measure). Assume that $a \in \mathscr{A}(H, M)$ (or $a \in \mathscr{A}(\Gamma, M)$ )

Received by the editors June 1, 1992 and, in revised form, November 12, 1993.

1991 Mathematics Subject Classification. Primary 57S20, 58F11.

(C)1994 American Mathematical Society 
is an ergodic action which preserves a volume. Then a sufficiently $C^{1}$-small perturbation $a^{\prime}$ of $a$ is conjugate to $a$ in $\operatorname{Diff}(M)$.

Local rigidity for the standard action of $\Gamma=S L(n, \mathbb{Z}) \subset S L(n, \mathbb{R})$ on $\mathbb{T}^{n}(n \geq 4)$ has been recently proved by A. Katok and J. Lewis [6, Theorem 1.3 , p. 2]. The example of the R-actions on the torus shows that local rigidity depends crucially on the group structure and not on the "richness" of the $H$-invariant geometric structure (since both the $a_{\alpha_{n}}$ and $a_{\alpha}$ leave the flat metric on $T^{2}$ invariant).

The actions dealt with in the Main Conjecture will be referred to as actions of type $a$. Canonical examples are obtained as follows: let $G=\Pi G_{i}$ be a finite product where each $G_{i}$ is a connected noncompact simple Lie group with finite center. Let $\Gamma \subset G$ be a cocompact lattice subgroup such that the transitive action of $G$ on $M=G / \Gamma$ is irreducible (we recall that if $M$ is a $G$-space with finite invariant volume, then the $G$-action is called irreducible if for every noncentral normal subgroup $N \subset G, N$ is ergodic on $M$ ). Let $H$ be a connected semisimple Lie group with finite center such that the rank of each simple factor is at least two, and let $h: H \rightarrow G$ be a homomorphism whose image $h(H) \subset G$ is closed and noncompact. By Moore's Ergodicity Theorem [1, Theorem 2.2.15, p. 21] the $H$-action on $M$ is ergodic, and thus of type $a$.

Statement of the main results. The Main Conjecture induces numerous "stability" properties for actions of type $a$. In particular if $a \in \mathscr{A}(H, M)$ is of type $a$, a $C^{1}$-small perturbation $a^{\prime}$ of $a$, being conjugate to $a$, is also of type $a$, and if we moreover assume that $a$ preserves a $G$-structure $\omega$ then $a^{\prime}$ must also preserve a $G$-structure of the same type. One might think of trying to prove such a stability result with $\omega$ a Riemannian metric on $M$, but we recall that the Lie algebra of a semisimple noncompact Lie group cannot be embedded into the Lie algebra of a compact Lie group, so that a connected semisimple noncompact Lie group $H$ cannot act faithfully on $M$ preserving a Riemannian metric. However, natural situations arise where $H$ preserves a foliation $\mathscr{F}$ on $M$ (i.e., $H$ permutes the leaves of the foliation) and a Riemannian metric on the leaves of this foliation: take for instance $M=(H \times G) / \Gamma$ where $G$ is a connected semisimple noncompact Lie group with finite center and $\Gamma \subset H \times G$ is a cocompact lattice (the leaf through $\overline{(h, g)}$ is then $\overline{(h, G)}$ on which we consider the $H$-invariant quotient of a right-invariant Riemannian metric on $G$ ). Such $H$-actions are moreover transverse to the foliation $\mathscr{F}$ (i.e., at each $m \in M$ we have $T_{m} M=T_{m} \mathscr{F}+T_{m}(\mathrm{Hm})$ ). The following theorem is thus clearly germane to the Main Conjecture, and one can actually show that it is implied by it.

Main Theorem (Theorem 4.1.1). Let $H$ be a connected semisimple Lie group with finite center which can be realized as the $\mathbf{R}$-points of an algebraic $\mathbf{R}$-group $\mathscr{H}$ and such that the rank of each simple factor is at least two. Assume that $a \in \mathscr{A}(H, M)$ is an irreducible $H$-action which preserves a smooth volume $v$, a foliation $\mathscr{F}$ and a smooth Riemannian metric $\omega$ on TF . Assume moreover that $a$ is transverse to $\mathscr{F}$. Let $a^{\prime}$ be a sufficiently $C^{\infty}$-small perturbation of $a$ which is irreducible, which preserves a smooth volume $v^{\prime}$ and the foliation $\mathscr{F}$. Then for each integer $0 \leq r<\infty$ there exists an $a^{\prime}$-invariant $C^{r}$-Riemannian metric on $T F$. 
It would be nice to generalize this theorem to $H$-actions which are not necessarily transverse to $\mathscr{F}$. A partial result in this direction is the following:

Theorem 3.1.1. Assume that $a \in \mathscr{A}(H, M)$ is an irreducible $H$-action ( $H$ satisfies the hypotheses of the Main Theorem) which preserves a smooth volume $v$, a foliation $\mathscr{F}$, and which leaves a continuous Riemannian metric $\omega$ on TF invariant. Let $a^{\prime}$ be a sufficiently $C^{1}$-small perturbation of $a$ which is irre-

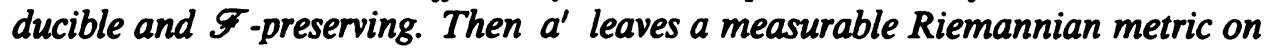
TF a.e. invariant.

Though the Main Conjecture implies that the irreducibility (or ergodicity) and the volume-preserving character of actions of type $a$ should be stable under small perturbations, as we have not succeeded proving such stability results, we assumed in the Main Theorem that the small perturbation $a^{\prime}$ was both irreducible and volume-preserving. However, in $\$ 2$, we prove the following theorem.

Theorem 2.2. Assume that $H$ is a Kazhdan Lie group (e.g., $H$ satisfies the hypotheses of the Main Conjecture) and that $a \in \mathscr{A}(H, M)$ preserves some probability $\mu \in M_{a}(M)$ (where $M_{a}(M)$ denotes the set of measures on $M$ which are absolutely continuous with respect to one, and hence any, smooth volume). Fix a smooth volume $v_{0}$ on $M$, an $\epsilon>0$, and let $a^{\prime} \in \mathscr{A}(H, M)$ be a sufficiently $C^{1}$-small perturbation of $a$. Then:

(1) $a^{\prime}$ preserves some probability $\mu^{\prime} \in M_{a}(M)$ satisfying

$$
\left\|\frac{d \mu^{\prime}}{d v_{0}}-\frac{d \mu}{d v_{0}}\right\|_{1, v_{0}} \leq 2 \epsilon .
$$

(2) If $\mu$ is a smooth volume we can moreover insure that $\mu^{\prime} \in M_{s}(M)$ (the smooth probability class on $M$ ).

Our Main Theorem is an analog of Zimmer's Theorem [2, Theorem 10.1, p. 192] which deals with isometric actions of lattice subgroups $\Gamma \subset H$ on $M$. Most of the material in this paper is part of my Ph.D. dissertation written at the University of Chicago under the guidance of Professor Robert J. Zimmer. I am grateful to him for suggesting this line of research and guiding my first steps in the research process.

\section{RIGIDITY OF THE VOLUME-PRESERVING CHARACTER OF ACTIONS OF TYPE $a$}

Throughout this section $H$ denotes a Kazhdan Lie group. We recall the corresponding definitions.

\section{Definitions 2.1.}

(1) Let $H$ be a Lie group and $(\Pi, \mathscr{H})$ be a unitary representation of $H$ on some Hilbert space $\mathscr{H}$. Let $\epsilon>0$ and $K \subset H$ a compact subset. $A$ unit vector $f \in \mathscr{H}$ is said to be $(\epsilon, K)$-invariant if $\|\Pi(h) f-f\| \leq \epsilon$ for each $h \in K$.

(2) We say that $\Pi$ almost has invariant vectors if for all $(\epsilon, K)$ there exists an $(\epsilon, K)$-invariant unit vector.

(3) The group $H$ is said to be Kazhdan if any unitary representation of $H$ which almost has invariant vectors actually has a nontrivial invariant vector. 
Let $M_{a}(M)$ denote the set of measures on $M$ which are absolutely continuous with respect to one (and hence any) smooth volume, and let $M_{s}(M)$ be the set of measures on $M$ which are in the same measure class as smooth volumes. As the Lie groups dealt with in the Main Conjecture 1.1 are Kazhdan $[1$, Theorem 7.1.4, p. 130], the following theorem, which is our first stability result, is clearly relevant to the Main Conjecture.

Theorem 2.2. Assume that $H$ is a Kazhdan Lie group and that $a \in \mathscr{A}(H, M)$ preserves some probability $\mu \in M_{a}(M)$. Fix a smooth volume $v_{0}$ and $\epsilon>0$. Let $a^{\prime} \in \mathscr{A}(H, M)$ be a sufficiently $C^{1}$-small perturbation of $a$. Then:

(1) $a^{\prime}$ preserves some probability $\mu^{\prime} \in M_{a}(M)$ satisfying

$$
\left\|\frac{d \mu^{\prime}}{d v_{0}}-\frac{d \mu}{d v_{0}}\right\|_{1, v_{0}} \leq 2 \epsilon .
$$

(2) If we moreover assume that $\mu$ is a smooth probability volume we can moreover insure that $\mu^{\prime} \in M_{s}(M)$.

We first construct a unitary representation $\Pi_{a^{\prime}}^{v_{0}}: H \rightarrow U\left(L_{v_{0}}^{2}(M)\right)$ : the $H$ action $a^{\prime}$ on $M$ induces an $H$-action on $M_{a}(M)$ defined by

$$
\left(a^{\prime}(h, \mu)\right)(A)=\mu\left(a^{\prime}\left(h^{-1}, A\right)\right), \quad A \subset M .
$$

By the Radon-Nikodym Theorem the map $\Phi_{v_{0}}: M_{a}(M) \rightarrow L_{v_{0}}^{2,+}(M)=\{f \in$ $\left.L_{v_{0}}^{2}(M) \mid f \geq 0\right\}$ defined by $\mu \mapsto\left(\frac{d \mu}{d v_{0}}\right)^{1 / 2}$ is a bijection so that the $H$-action $a^{\prime}$ on $M$ also yields through this bijection an $H$-action on $L_{v_{0}}^{2,+}(M)$. In order to find an explicit formula for this action we need to express $\left(\frac{d\left(a^{\prime}(h, \mu)\right)}{d v_{0}}\right)^{1 / 2}$ as a function of $\left(\frac{d \mu}{d v_{0}}\right)^{1 / 2}$. We have

$$
\frac{d\left(a^{\prime}(h, \mu)\right)}{d v_{0}}(m)=\frac{d \mu}{d v_{0}}\left(a^{\prime}\left(h^{-1}, m\right)\right) \frac{d\left(a^{\prime}\left(h, v_{0}\right)\right)}{d v_{0}}(m)
$$

and thus introduce the smooth strictly positive function

$$
J_{a^{\prime}}^{v_{0}}(h, m)=\left(\frac{d\left(a^{\prime}\left(h, v_{0}\right)\right)}{d v_{0}}(m)\right)^{1 / 2}
$$

and define the representation $\Pi_{a^{\prime}}^{v_{0}}: H \rightarrow U\left(L_{v_{0}}^{2}(M)\right)$ of $H$ on $L_{v_{0}}^{2}(M)$ by setting

$$
\left(\Pi_{a^{\prime}}^{v_{0}}(h) f\right)(m)=f\left(a^{\prime}\left(h^{-1}, m\right)\right) J_{a^{\prime}}^{v_{0}}(h, m) .
$$

By construction the representation $\Pi_{a^{\prime}}^{v_{0}}$ is unitary, $\mu \in M_{a}(M)$ is $a^{\prime}$-invariant if and only if $\left(\frac{d \mu}{d v_{0}}\right)^{1 / 2} \in L_{v_{0}}^{2}(M)$ is $\Pi_{a^{\prime}}^{v_{0}}$-invariant, and conversely, as $J_{a^{\prime}}^{v_{0}}>0$, if $f \in L_{v_{0}}^{2}(M)$ is. $\Pi_{a^{\prime}}^{v_{0}}$-invariant then $|f|$ is also $\Pi_{a^{\prime}}^{v_{0}}$-invariant and thus $\mu=$ $f^{2} v_{0} /\|f\|_{2, v_{0}}^{2}$ is an $a^{\prime}$-invariant probability on $M$.

Proof of Theorem 2.2. Let $f=\left(\frac{d \mu}{d v}\right)^{1 / 2}$ be the $\Pi_{a}^{v_{0}}$-invariant function in $L_{v_{0}}^{2}(M)$ associated to the $a$-invariant probability $\mu$, let $\left(a_{n}\right)_{n \in N}$ be a sequence of $H$-actions which converge to $a$ in the topology of $C^{1}$-uniform convergence on compact subsets of $H$, and let $\left(\Pi_{a_{n}}^{v_{0}}\right)_{n \in N}$ be the associated sequence of unitary representations on $L_{v_{0}}^{2}(M)$. 
We first prove (1), namely that for $n$ large enough there exist $\Pi_{a_{n}}^{0}$-invariant unit functions $f_{n}$ in $L_{v_{0}}^{2}(M)$ which satisfy $\left\|f_{n}-f\right\|_{2, v_{0}} \leq \epsilon$ (as indeed if we then set $\mu_{n}=f_{n}^{2} v_{0}$ we do have $\left\|\frac{d \mu_{n}}{d v_{0}}-\frac{d \mu}{d v_{0}}\right\|_{1, v_{0}}=\int_{M}\left|f_{n}^{2}-f^{2}\right| d v_{0}=$ $\int_{M}\left|f_{n}+f\left\|f_{n}-f \mid d v_{0} \leq\right\| f_{n}+f\left\|_{2, v_{0}}\right\| f_{n}-f \|_{2, v_{0}} \leq 2 \epsilon\right)$. It is well known that the definition of a Kazhdan group can be reformulated as follows.

Lemma 2.3 [5, Proposition 16, p. 15]. There exist a compact subset $K_{0} \subset H$ (depending only on $H$ ) and $a \delta>0$ (depending only on $\epsilon$ and $H$ ) such that for any unitary representation $(\Pi, \mathscr{H})$ of $H$ on a Hilbert space $\mathscr{H}$ and given any $\left(\delta, K_{0}\right)$-invariant unit vector $f \in \mathscr{H}$ there exists an $H$-invariant unit vector $g \in \mathscr{H}$ with $\|g-f\|<\epsilon$.

Let $\delta>0$ and $K_{0} \subset H$ as in Lemma 2.3: it is enough, by this lemma, to show that $f$ is a $\left(\delta, K_{0}\right)-\Pi_{a_{n}}^{v_{0}}$-invariant vector for $n$ large enough. But if we set

$$
A=\sup _{\left[m \in M, n \in \mathbb{N}, h \in K_{0}\right]} J_{a_{n}}^{v_{0}}(h, m)
$$

we have:

$$
\begin{aligned}
\left\|\Pi_{a_{n}}^{v_{0}}(h) f-f\right\|_{2, v_{0}} & =\left\|\left[\Pi_{a_{n}}^{v_{0}}(h)-\Pi_{a}^{v_{0}}(h)\right] f\right\|_{2, v_{0}} \\
& \leq A\left\|f\left(a_{n}\left(h^{-1}, .\right)\right)-f\left(a\left(h^{-1}, .\right)\right)\right\|_{2, v_{0}}
\end{aligned}
$$

which converges uniformly on $K_{0}$ to 0 . This proves (1).

In order to prove (2) we can assume without loss of generality that $\mu=v_{0}$ (so that the asociated $\Pi_{a}^{v_{0}}$-invariant function in $L_{v_{0}}^{2}(M)$ is $f=1$, and $J_{a}^{v_{0}}=$ 1). There exists an integer $n_{0}$ such that for all $n \geq n_{0}$ and all $h \in K_{0}$, $\left\|\Pi_{a_{n}}^{v_{0}}(h) 1-1\right\|_{2, v_{0}}=\left\|J_{a_{n}}^{v_{0}}(h, .) 1-1\right\|_{2, v_{0}} \leq\left\|J_{a_{n}}^{v_{0}}(h, .)-1\right\|_{\infty}<\delta$ (the compact $K_{0} \subset H$ and $\delta>0$ are again yielded by Lemma 2.3).

From now on we fix such an integer $n \geq n_{0}$ and show that there exists an $a_{n}$-invariant $\mu_{n} \in M_{s}(M)$.

Again let $g$ be the $\Pi_{a_{n}}^{v_{0}}$-invariant function in $L_{v_{0}}^{2}(M)$ satisfying $\|g-1\|_{2, v_{0}}$ $<\epsilon$ yielded by Lemma 2.3. By replacing $g$ by $|g|$ we can assume without loss of generality that $g \geq 0$. We would like to have the additional property $g>0$. Set $Z_{g}=\{m \in M \mid g(m)=0\}$. As $J_{a_{n}}^{v_{0}}>0, Z_{g}$ is $a_{n}$-invariant. As $\|g-1\|_{2, v_{0}}<\epsilon$, necessarily $v_{0}\left(Z_{g}\right)<\epsilon^{2}$. If $v_{0}\left(Z_{g}\right)=0$ we are done. Assume thus that $Z_{g}$ is not a null set. We can then consider the "restricted representation" $\Pi_{a_{n}}^{v^{\prime}}: H \times L_{v^{\prime}}^{2}\left(Z_{g}\right) \rightarrow L_{v^{\prime}}^{2}\left(Z_{g}\right)$ which is unitary (we set $v^{\prime}=v_{0} / v_{0}\left(Z_{g}\right)$ ). But 1 is again a $\left(\delta, K_{0}\right)-\Pi_{a_{n}}^{v^{\prime}}$-invariant vector so that there exists a unit $g_{1} \in$

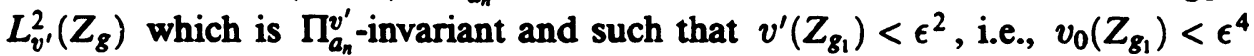
and $\left\|1-g_{1}\right\|_{2, v^{\prime}}<\epsilon$. Proceed then inductively: we obtain a decreasing sequence of measurable sets $Z_{g} \supset Z_{g_{1}} \supset Z_{g_{2}} \supset \ldots \supset Z_{g_{k}} \supset \ldots$ such that $v\left(Z_{g_{k}}\right)<\epsilon^{2 k}$ together with a sequence of functions $(M, g),\left(Z_{g}, g_{1}\right),\left(Z_{g_{1}}, g_{2}\right), \ldots$. Extend $g_{i}$ on $M$ by 0 and set $\phi=g+\sum_{1}^{\infty} g_{k}$. By construction $\phi$ is $\prod_{a_{n}}^{v_{0}}$-invariant, $\phi \in L_{v_{0}}^{2}(M),\|\phi-1\|_{2, v_{0}}<2 \epsilon$, and $\phi>0$. Thus $\mu_{n}=\phi^{2} v_{0} \in M_{s}(M)$ is an $a_{n}$-invariant probability which is solution of our problem.

We end this section with an example of a non-Kazhdan Lie group for which the conclusion of Theorem 2.2 fails: let $H=\left(\mathbf{R}_{+}^{*}, \times\right)$, let $M=S^{2} \subset \mathbf{R}^{3}$ be the 2-sphere and $a: H \times S^{2} \rightarrow S^{2}$ be the natural $H$-action on $S^{2}$ induced by 
the rotation of $\mathbb{R}^{3}$ around the vertical axis with angle $\ln h$, which preserves the canonical volume of $S^{2}$. For any $h>0$ let $\Phi_{h}$ be the diffeomorphism of $S^{2}$ that fixes the poles and which, viewed in the chart of the stereographic projection through the North pole (resp. the South pole) is a homothety of ratio $h$ (resp. $1 / h)$ and set

$$
a_{k}(h)=\Phi_{h^{1 / k}} \circ R_{\ln h} .
$$

Though the sequence of $H$-actions $a_{k}$ converges to $a$ in the topology of $C^{\infty}$. uniform convergence on compact subsets of $H$, it is easy to see that none of the $a_{k}$ leaves a measure in $M_{a}(M)$ invariant.

\section{RIGIDITY OF THE MEASURABLE METRIC PRESERVING CHARACTER OF ACTIONS OF TYPE $a$}

3.1. Introduction. In this section we prove a stability result by applying the Superrigidity Theorem for Cocycles (Theorem 3.3.3), for which we need to assume that $H=\mathscr{H}_{\mathrm{R}}^{0}$ where $\mathscr{H}$ is a connected semisimple algebraic R-group such that each simple factor has R-rank at least 2: from now on we make this additional assumption on $H$. We recall that if $H$ satisfies the hypotheses of the Main Conjecture and is moreover center-free, then this additional assertion is redundant. As we said in the introduction, natural situations arise where such groups $H$ act on a compact manifold $M$ preserving a foliation and a Riemannian metric on the leaves of this foliation:

Theorem 3.1.1. Assume that $H=\mathscr{X}_{\mathbf{R}}^{0}$ where $\mathscr{H}$ is a connected semisimple algebraic R-group such that each simple factor has R-rank at least 2. Let a $\in$ $\mathscr{A}(H, M)$ be an irreducible $H$-action which preserves a smooth volume $v, a$ foliation $\mathscr{F}$, and which leaves a continuous Riemannian metric $\omega$ on $T \mathscr{F}$ invariant. Let $a^{\prime}$ be a sufficiently $C^{1}$-small perturbation of a which is irreducible and $\mathscr{F}$-preserving. Then $a^{\prime}$ leaves a measurable Riemannian metric on $T \mathscr{F}$ a.e. invariant.

As Theorem 2.2 asserts that if $a^{\prime}$ is a sufficiently $C^{1}$-small perturbation of $a$, then there exists an $a^{\prime}$-invariant $\mu^{\prime} \in M_{s}(M)$, we can assume without loss of generality that the perturbed action $a^{\prime}$ we consider leave some $\mu^{\prime} \in M_{s}(M)$ (depending on $a^{\prime}$ ) invariant. From now on we furthermore tacitly assume that the $H$-actions considered are irreducible and $\mathscr{F}$-preserving.

3.2. The algebraic hull of the ergodic $H$-action $a^{\prime}$. We will assume that the reader is familiar with the machinery of cocycles applied to measurable reductions of principal bundles as exposed, for instance, in [3, $\S 2, \mathrm{pp} .251-256]$. Set $d=\operatorname{dim}(M)$ and $p=\operatorname{dim}(\mathscr{F})$. Let $P$ be the $G L(p)$-principal fiber bundle of the frames of $\mathscr{F}$. As the $H$-action $a^{\prime}$ on $M$ is assumed to be $\mathscr{F}$-preserving, it lifts to an $H$-action on $P$ by principal fiber bundle automorphisms, and as the $H$-action $a^{\prime}$ on $M$ is assumed to be ergodic, one can canonically attach to $a^{\prime}$ an algebraic subgroup of $G L(p)$ called the algebraic hull of the $H$-action $a^{\prime}$ : let $s: M \rightarrow P$ be a measurable section of $P$ and let $\Phi: M \times G L(p) \rightarrow P$ denote the corresponding measurable trivialization (defined by $\Phi(m, g)=s(m) g$ ). As $H$ acts on $P$ by principal bundle automorphisms we can describe the $H$-action on $P$ in the trivialization $\Phi$ by the cocycle $\alpha: H \times M \rightarrow G L(p)$ (defined by $h . \Phi(m, g)=\Phi(h m, \alpha(h, m) g))$. 
Theorem 3.2.1 (algebraic hull of a cocycle) [3, Theorem 4.1, p. 260]. Let $\alpha$ : $H \times M \rightarrow G L(p)$ be a cocycle. Then,

(1) There exists an algebraic $\mathbf{R}$-group $\mathscr{L} \subset G L(p, \mathbb{C})$ such that $\alpha$ is equivalent to a cocycle taking values in $L=\mathscr{L}_{\mathbb{R}}$ but $\alpha$ is not equivalent to a cocycle taking values in a proper subgroup of $L$ of the form $M=M_{\mathbb{R}}$ where $\mathscr{M}$ is also an algebraic R-group.

(2) $L$ is unique up to conjugacy. The conjugacy class of $L$ (or by abuse of language $L$ or $\mathscr{L}$ ) is called the algebraic hull of the cocycle $\alpha$.

(3) If $\alpha \sim \beta$ where $\beta(H \times M) \subset L^{\prime}$ and $L^{\prime}=\mathscr{L}_{\mathbf{R}}^{\prime}$ where $\mathscr{L}^{\prime}$ is an algebraic $\mathbb{R}$-group then $L^{\prime}$ contains a conjugate of $L$.

Remark. Since $a^{\prime}$ canonically defines an equivalence class of cocycles, it does make sense to speak of the algebraic hull of the $H$-action $a^{\prime}$. We will also use the following results:

Theorem 3.2.2.

(1) $[3, \S 2]$ There exists an a.e. $a^{\prime}$-invariant measurable metric on $T \mathscr{F}$ if and only if $L$ is compact.

(2) $[4$, Theorem 1.1, p. 375$] L$ is a reductive group with compact center (this uses essentially the hypotheses we made on $H$ ).

3.3. $H$-actions with noncompact algebraic hulls. Fix a continuous Riemannian

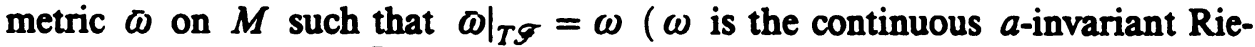
mannian metric on $T \mathscr{F}$ ). By abuse of notation we will write $\omega=\omega$ and we denote by \|\| the associated norm on $T M$. In this section we fix an $H$-action $a^{\prime}$ on $M$ with noncompact algebraic hull (which preserves some $\mu^{\prime} \in M_{s}(M)$ with respect to which the action is irreducible) and prove

Theorem 3.3.1. There exist an $h_{0} \in H$ and $a \lambda>1$, both independent of the fuxed action considered (with noncompact algebraic hull), $a C>0$ and a vector $v \in T \mathscr{F}$, such that for infinitely many integers $n$ we have $\left\|h_{0}^{n} v\right\| \geq C \lambda^{n}$.

Let $L=\mathscr{L}_{R}$ be the algebraic hull of the $H$-action on $P$. There exists a trivialization $\Phi_{0}: M \times G L(p) \rightarrow P$ of $P$ (corresponding to the measurable section $s_{0}(m)=\Phi_{0}(m, e)$ ) in which the action is described by the cocycle $\alpha: H \times M \rightarrow L$. From now on we will work in this trivialization.

Lemma 3.3.2. There exists a finite irreducible extension $p: \widetilde{M} \rightarrow M$ (i.e., all fibers are of a fixed finite cardinality), a nontrivial homomorphism $\tilde{\rho}: \widetilde{H} \rightarrow$ $\left(\mathscr{L}^{0}\right)_{\mathbb{R}}$ (where $(\tilde{H}, \pi)$ denotes the universal covering of $H$ ), a compact normal subgroup $K \triangleleft\left(\mathscr{L}^{0}\right)_{\mathbf{R}}$, a measurable map $\Phi: \widetilde{M} \rightarrow\left(\mathscr{L}^{0}\right)_{\mathbf{R}}$, and a map $\Psi$ : $H \times \widetilde{M} \rightarrow K$, such that for all $h \in H$ and any $\widetilde{h} \in \widetilde{H}$ satisfying $\pi(\widetilde{h})=h$, we have

$$
\alpha(h, m)=\Phi^{-1}(h \tilde{m}) \tilde{p}(\tilde{h}) \Phi(\tilde{m}) \Psi(h, \tilde{m})
$$

for a.e. $m \in M$ and any $\tilde{m} \in \widetilde{M}$ such that $p(\tilde{m})=m$.

Proof. By [1, Proposition 9.2.6, p. 168] there exists a finite irreducible extension $p: \widetilde{M} \rightarrow M$ such that the cocycle defined by

$$
\tilde{\alpha}(h, \tilde{m})=\alpha(h, p(\tilde{m}))
$$


has algebraic hull $\left(\mathscr{L}^{0}\right)_{\mathbf{R}}$. As $\left(\mathscr{L}^{0}\right)_{\mathbf{R}} \subset L$ has finite index and $L$ is assumed to be noncompact, $\left(\mathscr{L}^{0}\right)_{\mathrm{R}}$ is noncompact. As $Z(L)=(Z(\mathscr{L}))_{\mathrm{R}}$ is compact (by (2) of Theorem 3.2.2), $Z\left(\left(\mathscr{L}^{0}\right)_{\mathrm{R}}\right)$ is compact. As $\mathscr{L}$ is a reductive algebraic R-group (by (2) of Theorem 3.2.2), $\mathscr{L}^{0}$ is a connected reductive algebraic $\mathbf{R}$ group so that we can write

$$
\mathscr{L}^{0} / Z\left(\mathscr{L}^{0}\right)=\mathscr{L}_{1} \times \mathscr{L}_{2}
$$

where both $\mathscr{L}_{1}$ and $\mathscr{L}_{2}$ are connected center-free semisimple algebraic $\mathbb{R}$ groups such that $L_{1}=\left(\mathscr{L}_{1}\right)_{\mathrm{R}}$ is a compact semisimple Lie group, $\mathscr{L}_{2}=\mathscr{S}_{1} \times$ $\cdots \times \mathscr{S}_{n}$ where each $\mathscr{S}_{i}$ is a connected simple center-free algebraic R-group such that each $S_{i}=\left(\mathscr{S}_{i}\right)_{\mathrm{R}}$ is a simple, center-free noncompact Lie group. Let $q$ and $q_{i}$ be the canonical homomorphisms of $\mathbf{R}$-group defined over $\mathbf{R}$,

$$
q: \mathscr{L}^{0} \rightarrow \mathscr{L}^{0} / Z\left(\mathscr{L}^{0}\right) \rightarrow \mathscr{L}_{2}, \quad q_{i}: \mathscr{L}_{2} \rightarrow \mathscr{S}_{i},
$$

and set

$$
\widetilde{\alpha}_{i}=q_{i} \circ q \circ \tilde{\alpha}: H \times \widetilde{M} \rightarrow S_{i} .
$$

The cocycle $\tilde{\alpha}_{i}$ is not equivalent to a cocycle taking values in a proper subgroup of the form $G_{i}=\left(\mathscr{G}_{i}\right)_{\mathrm{R}}$ where $\mathscr{G}_{i} \subset \mathscr{S}_{i}$ is an algebraic $\mathbf{R}$-subgroup (otherwise $\tilde{\alpha}$ would be equivalent to a cocycle taking values in $\left[\left(q_{i} \circ q\right)^{-1}\left(\mathscr{G}_{i}\right)\right]_{\mathrm{R}}$ where $\left(q_{i} \circ q\right)^{-1}\left(\mathscr{G}_{i}\right) \subset \mathscr{L}^{0}$ is a proper algebraic $\mathbf{R}$-subgroup, which is impossible as $\left(\mathscr{L}^{0}\right)_{\mathrm{R}}$ is the algebraic hull of the cocycle $\left.\tilde{\alpha}\right)$, so that we can apply the following theorem with $\mathscr{S}=\mathscr{S}_{i}$ and $\beta=\tilde{\alpha}_{i}$ :

3.3.3. Superrigidity Theorem for Cocycles [1, Theorem 5.2.5, p. 98]. Suppose that $\mathscr{H}$ is a connected semisimple algebraic $\mathbf{R}$-group such that $\mathbf{R}-\operatorname{rank}(H) \geq 2$ and $H=\mathscr{K}_{\mathbf{R}}^{0}$ has no compact factors. Suppose that $\widetilde{M}$ is an irreducible $H$ space with a finite invariant measure $\tilde{\mu}$. Let $\mathscr{S}$ be a connected simple algebraic R-group, set $S=\mathscr{S}_{\mathbb{R}}$ and suppose that $\beta: H \times \widetilde{M} \rightarrow S$ is a cocycle such that $\beta$ is not equivalent to a cocycle taking values in a subgroup $G=(\mathscr{E})_{\mathrm{B}}$ where $\mathscr{G} \subset \mathscr{S}$ is a proper algebraic $\mathbf{R}$-subgroup. Then if $S$ is not compact, there exists a rational homomorphism of $\mathbf{R}$-groups $\rho: \mathscr{H} \rightarrow \mathscr{S}$ defined over $\mathbf{R}$ and such that $\beta \sim \alpha_{\left.\rho\right|_{H}}$ where $\alpha_{\left.\rho\right|_{H}}(h, \tilde{m})=\left.\rho\right|_{H}(h)$.

Thus for each $i=1, \ldots, n$ there exists a rational homomorphism of $\mathbf{R}$ groups $\rho_{i}: \mathscr{H} \rightarrow \mathscr{S}_{i}$ defined over $\mathbf{R}$ and a measurable $\widetilde{\phi}_{i}: \widetilde{M} \rightarrow S_{i}$ such that for all $h \in H$ we have $\tilde{\alpha}_{i}(h, \tilde{m})=\left.\phi_{i}^{-1}(h m) \rho_{i}\right|_{H}(h) \phi_{i}(m)$ for a.e. $\tilde{m} \in \widetilde{M}$. Equivalently there exists a measurable $\phi: \widetilde{M} \rightarrow L_{2}$ and a rational homomorphism of R-groups $\rho: \mathscr{H} \rightarrow \mathscr{L}_{2}$ defined over $\mathbf{R}$ such that for all $h \in H$ we have

$$
q \circ \tilde{\alpha}(h, \tilde{m})=\left.\phi^{-1}(h \tilde{m}) \rho\right|_{H}(h) \phi(\tilde{m}) \text { for a.e. } \tilde{m} \in \widetilde{M}
$$

where $\left.\rho\right|_{H}: H=\mathscr{X}_{\mathbf{R}}^{0} \rightarrow L_{2}^{0}$ (as we assume $H$ is connected). Let $d \rho: \mathfrak{h} \rightarrow \mathfrak{l}_{2}$ denote the Lie algebra homomorphism corresponding to $\left.\rho\right|_{H}$ and let $\mathfrak{l}$ be the Lie algebra of $\left(\mathscr{L}^{0}\right)_{\mathbb{R}}$. As $\mathfrak{h}$ is semisimple we can lift $d \rho$ to $d \tilde{\rho}: \mathfrak{h} \rightarrow \mathfrak{l}$ and thus there exists a homomorphism of Lie groups $\tilde{\rho}: \widetilde{H} \rightarrow\left(\mathscr{L}^{0}\right)_{\mathrm{R}}^{0}$ corresponding to $d \tilde{\rho}: \mathfrak{h} \rightarrow \mathfrak{l}$ and covering $\left.\rho\right|_{H}: H \rightarrow L_{2}^{0}$. Fix $r: L_{2}^{0} \rightarrow\left(\mathscr{L}^{0}\right)_{\mathbb{R}}^{0}$ a measurable 
section of $q$ and set $\Phi=r \circ \phi$. The following diagram commutes:

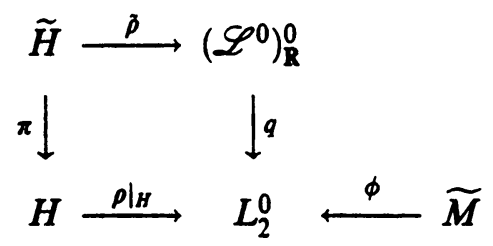

Let $K \triangleleft\left(\mathscr{L}^{0}\right)_{\mathrm{R}}$ be the normal Lie subgroup $K=q^{-1}(e)$. The compactness of $L_{1}$ and $\left(Z\left(\mathscr{L}^{0}\right)\right)_{\mathrm{R}}$ implies that $K$ is compact. Let $\tilde{h} \in \tilde{H}$ such that $\pi(\tilde{h})=h$. As $q(a)=q(b) \Leftrightarrow a=b k$ for some $k \in K$ and $q\left(\Phi^{-1}(h \tilde{m}) \tilde{p}(\tilde{h}) \Phi(\tilde{m})\right)=$ $\left.\phi^{-1}(h \tilde{m}) \rho\right|_{H}(h) \phi(\tilde{m})=q \circ \tilde{\alpha}(h, \tilde{m})$ we have, for all $\tilde{h} \in \tilde{H}$,

$$
\tilde{\alpha}(h, \tilde{m})=\Phi^{-1}(h \tilde{m}) \tilde{\rho}(\tilde{h}) \Phi(\tilde{m}) \Psi(h, \tilde{m})
$$

for a.e. $\tilde{m} \in \widetilde{M}$ and for some $\Psi: H \times \widetilde{M} \rightarrow K$. Clearly $\tilde{\rho}$ cannot be trivial (otherwise $\tilde{\alpha}$ and thus $\alpha$ would be equivalent to the cocycle $\Psi(h, \tilde{m})$ which takes its values in $K$ which is compact so that the algebraic hull of the $H$-action $L$ would be compact). As $\alpha(h, m)=\tilde{\alpha}(h, \tilde{m})$ we are done.

At each $m \in M$ the measurable section $s_{0}: M \rightarrow P$ defines a canonical linear isomorphism $s_{0}(m): \mathbf{R}^{p} \rightarrow T_{m} F$. Let \|\|$_{R^{p}}$ denote the usual norm on $\mathbf{R}^{p}$ and $\pi: T \mathscr{F} \rightarrow M$ be the canonical projection. The following lemma is an easy exercise.

Lemma 3.3.4. There exists a measurable $\tilde{A} \subset \widetilde{M}$ with $\tilde{\mu}(\tilde{A})>0$ and $a C^{\prime}>0$ satisfying.

(1) $\|v\| \geq C^{\prime}\left\|s_{0}(\pi(v))^{*} v\right\|_{\mathbb{R}^{p}}$ for all $v \in \pi^{-1}(p(\tilde{A})$ ) (where $p: \widetilde{M} \rightarrow M$ is the finite irreducible extension introduced in Lemma 3.3.2),

(2) $\Phi(\tilde{A}) \subset K^{\prime}$ for some compaci $K^{\prime} \subset L$ (where $\Phi$ is the map introduced in Lemma 3.3.2).

Lemma 3.3.5 (Poincaré recurrence) [1, Lemma 9.1.5, p. 165]. Let $(X, \mu)$ be an $H$-space and assume that $\mu$ is $H$-invariant. Fix $h \in H$ and $A \subset X$ such that $\mu(A) \neq 0$. Then for almost all $x \in A, h^{n} x \in A$ for infinitely many positive $n$.

Proof of Theorem 3.3.1. Let $\Pi=\tilde{\rho}: \tilde{H} \rightarrow\left(\mathscr{L}^{0}\right)_{\mathrm{R}}^{0} \subset L \subset G L(p)$ be the canonical representation of $\tilde{H}$ on $\mathbf{R}^{p}$ associated to $\tilde{\rho}$. As $\tilde{H}$ is a simply connected semisimple Lie group such that the rank of each simple factor is greater than 1 (positive would actually be enough for what follows), there exists an $\tilde{h}_{0} \in \tilde{H}$ and a $\lambda>1$, both depending only on $\tilde{H}$, a $\xi \in \mathbb{R}^{p}$ and a $\beta \in \mathbf{R}$ with $|\beta|>\lambda$ such that $\tilde{\rho}\left(\tilde{h}_{0}\right) \xi=\beta \xi$. Let $h_{0}=\pi\left(\tilde{h}_{0}\right)$ and let $\tilde{A} \subset \widetilde{M}$ as in Lemma 3.3.4. By Lemma 3.3 .5 we can pick an $\tilde{m} \in \widetilde{M}$ such that $h_{0}^{n} \tilde{m} \in \tilde{A}$ for infinitely many integers $n$ and the following diagram commutes (again set $m=p(\tilde{m})$ ):

$$
\begin{array}{cccccc}
T_{s_{0}(m)} \mathscr{F} & \stackrel{h_{0}}{\longrightarrow} & T_{s_{0}\left(h_{0} m\right)} \mathscr{F} & \stackrel{h_{0}}{\longrightarrow} & T_{s_{0}\left(h_{0}^{2} m\right)} \mathscr{F} \stackrel{h_{0}}{\longrightarrow} T_{s_{0}\left(h_{0}^{3} m\right)} \mathscr{F} \\
\uparrow_{s_{0}(m)} & \uparrow_{s_{0}\left(h_{0} m\right)} & \uparrow_{s_{0}\left(h_{0}^{2} m\right)} & \uparrow_{s_{0}\left(h_{0}^{3} m\right)} \\
\mathbf{R}^{p} & \stackrel{\alpha\left(h_{0}, m\right)}{\longrightarrow} & \mathbf{R}^{p} & \stackrel{\alpha\left(h_{0}, h_{0} m\right)}{\longrightarrow} & \mathbf{R}^{p} \stackrel{\alpha\left(h_{0}, h_{0}^{2} m\right)}{\longrightarrow} & \mathbf{R}^{p}
\end{array}
$$


Set $v=s_{0}(m) *\left((\Phi(\tilde{m}))^{-1} \xi\right) \in T_{m} \mathscr{F}$ where $\Phi: \widetilde{M} \rightarrow L \subset G L(p)$ is the map introduced in Lemma 3.3.2. By Lemma 3.3.2 we have

$$
\begin{aligned}
s_{0}\left(h_{0}^{n} m\right)^{*}\left(h_{0}^{n} v\right) & =\alpha\left(h_{0}^{n}, m\right)\left((\Phi(\tilde{m}))^{-1} \xi\right) \\
& =k_{n}\left(\Phi\left(h_{0}^{n} \tilde{m}\right)\right)^{-1} \tilde{\rho}\left(\tilde{h}_{0}^{n}\right) \xi=k_{n}\left(\Phi\left(h_{0}^{n} \tilde{m}\right)\right)^{-1} \beta^{n} \xi
\end{aligned}
$$

for some sequence $\left(k_{n}\right)_{n \in \mathbb{N}}$ of elements of $K$ (we recall that $K \triangleleft\left(\mathscr{L}^{0}\right)_{\mathbf{R}}$ ). By Lemma 3.3.4 this implies that for infinitely many integers $n$ we have

$$
\left\|h_{0}^{n} v\right\| \geq C^{\prime}\left\|k_{n}\left(\Phi\left(h_{0}^{n} \tilde{m}\right)\right)^{-1} \beta^{n} \xi\right\|_{\mathbb{R}^{p}} \geq C|\beta|^{n}
$$

for some constant $C>0$ and thus that for those integers we have $\left\|h_{0}^{n} v\right\| \geq$ $C \lambda^{n}$.

3.4. Proof of Theorem 3.1. The proof goes by contradiction: assume that there exists a sequence $\left(a_{k}\right)_{k \in \mathrm{N}}$ of $H$-actions on $M$ with noncompact algebraic hulls which converges to $a$ in the $C^{1}$-topology of uniform convergence on compact subsets of $H$. Let $h_{0} \in H$ and $\lambda>1$ be yielded by Theorem 3.3.1 and fix $\gamma \in \mathbf{R}$ such that $1<\gamma<\lambda$.

As the sequence $\left(a_{k}\right)_{k \in \mathbb{N}}$ converges to $a$ and as $a$ preserves the continuous norm \|\| , there exists an integer $k_{0} \in \mathbb{N}$ such that

$$
k \geq k_{0} \Rightarrow \text { for all } v \in T \mathscr{F}, \quad\left\|a_{k}\left(h_{0}, v\right)\right\| \leq \gamma\|v\| .
$$

We now fix an integer $k \geq k_{0}$ : by Theorem 3.3.1 there exist a $C>0$ and a $v \in T \mathscr{F}$ (which we can assume to be unitary) such that for infinitely many integers $n$ we have

$$
\left\|a_{k}\left(h_{0}^{n}, v\right)\right\| \geq C \lambda^{n} .
$$

But by $(*)$ we have $\left\|a_{k}\left(h_{0}^{n}, v\right)\right\| \leq \gamma^{n}$, so that by combining (*) and (**) we get $0<C \leq\left(\frac{y}{\lambda}\right)^{n}$ for infinitely many integers $n$, which is impossible.

\section{THE MAIN THEOREM}

4.1. Introduction. We now assume that the original $H$-action $a$ on $M$ leaves a smooth Riemannian metric $\omega$ on $T \mathscr{F}$ invariant. Let $a^{\prime}$ be a sufficiently $C^{\infty}$-small perturbation of $a$. In this section we prove the existence, for each integer $r$, of an $a^{\prime}$-invariant $C^{r}$-Riemannian metric on $T \mathscr{F}$. We will need the additional assumption that the original $H$-action $a$ is transverse to the foliation $\mathscr{F}$, namely that at each $m \in M$ we have $T_{m} \mathscr{F}+T_{m}(H m)=T_{m} M$.

4.1.1. Main Theorem. Let $H$ be a connected semisimple Lie group with finite center which can be realized as the $\mathbb{R}$-points of an algebraic $\mathbf{R}$-group $\mathscr{H}$ and such that the rank of each simple factor is at least two. Assume that $a \in \mathscr{A}(H, M)$ is an irreducible $H$-action which preserves a smooth volume $v$, a foliation $\mathscr{F}$, and a smooth Riemannian metric $\omega$ on TF. Assume moreover that $a$ is transverse to $\mathscr{F}$. Let $a^{\prime}$ be a sufficiently $C^{\infty}$-small perturbation of a which is irreducible, which preserves a smooth volume $v^{\prime}$ and the foliation $\mathscr{F}$. Then for each integer $0 \leq r<\infty$ there exists an $a^{\prime}$-invariant $C^{r}$-Riemannian metric on TF.

By Theorem 3.1.1, if $a^{\prime}$ is a sufficiently $C^{1}$-small perturbation of $a$, then $a^{\prime}$ leaves a measurable Riemannian metric on $T \mathscr{F}$ a.e. invariant; it is moreover 
easy to see that the transversality of $a$ implies the transversality of $C^{1}$-nearby actions: from now on $a^{\prime}$ will denote an irreducible $H$-action on $M$ which preserves the foliation $\mathscr{F}$, a smooth volume $v^{\prime}$ (depending on $a^{\prime}$ ), and which is contained in a sufficiently small $C^{1}$-neighbourhood of a so that it is transverse to $F$ and leaves a measurable Riemannian metric on $T \mathscr{F}$ a.e. invariant.

Sketch of the proof of the Main Theorem. In $\$ \$ 4.2,4.3$, and 4.4 we build the machinery needed to show that if the perturbation $a^{\prime}$ is sufficiently $C^{\infty}$-close to $a$, then for each integer $r$ there exists a nontrivial $\tilde{a}^{\prime}$-invariant function in $L^{2}(P) \cap C^{r}(P)$ (where again $P$ denotes the $G L(p)$-bundle of the frames of the foliation $F$ and $\tilde{a}^{\prime}$ denotes the $H$-action on $P$ by principle bundle automorphisms induced by the $H$-action $a^{\prime}$ on $M$ ). In $\S 4.5$ we show how the existence of such a function on $P$ implies the existence of an $a^{\prime}$-invariant $C^{r}$-Riemannian metric on $T \mathscr{F}$.

Remark. As $\S \S 4.2,4.3$, and 4.4 mimic $\S \S 2,4$, and 8 of [2] we adopt a terse style and refer the reader to Professor Zimmer's original paper for details or proofs. However, the techniques involved in $\$ 4.5$ differ significantly from the ones in [2] (which are inapplicable here as we do not have compactness of the isometry group $\operatorname{Is}(M, \omega))$ : we develop them in full details.

4.2. The metrics $\tilde{\omega}_{k}$ and $\widetilde{\eta_{k}^{\prime}}$ on the vector bundles $J_{\widetilde{g}}^{k}(P, \mathbf{R})$. Let $P=$ $F(\mathscr{F})$ denote the $G L(p)$-bundle of frames of the foliation $\mathscr{F}, \pi$ the projection $P \rightarrow M$, and let $\mathscr{D}$ denote the $p$-dimensional integrable distribution on $M$ which yields the foliation $\mathscr{F}$. If $u \in P$ and $m=\pi(u)$, define $\widetilde{\mathscr{D}}_{u}=\pi^{-1}\left(\mathscr{D}_{m}\right) \subset T_{u}(P)$. This yields a $\left(p+p^{2}\right)$-dimensional distribution $\widetilde{\mathscr{D}}$ on $P$ which is $G L(p)$-invariant and integrable: let $\widetilde{F}$ denote the corresponding $G L(p)$-invariant foliation. It is easy to see that an $H$-action on $M$ which is transverse to $\mathscr{F}$ (and $\mathscr{F}$-preserving) lifts to an $H$-action on $P$ transverse to $\widetilde{F}$ (and $\widetilde{F}$-preserving). Let $\operatorname{Met}(T \mathscr{F}) \rightarrow M$ and $\operatorname{Met}(T \widetilde{\mathscr{F}}) \rightarrow P$ denote the bundles whose sections are smooth Riemannian metrics on $T \mathscr{F}$ and $T \widetilde{F}$ respectively. The following proposition enables us to lift metrics on $T \mathscr{F}$ to metrics on $T \widetilde{\mathscr{F}}$ :

Proposition 4.2.1. There exists a natural bundle map from $\operatorname{Met}(T \mathscr{F})$ into $\operatorname{Met}(T \widetilde{\mathscr{F}})$ sending $\xi$ to $\tilde{\xi}$ such that:

(1) the Riemannian metric $\tilde{\xi}$ is $G L(p)$-invariant,

(2) the map $\xi \mapsto \tilde{\xi}$ is continuous, where $\operatorname{Met}(T \mathscr{F})$ has the topology of $C^{l}$. uniform convergence on compact sets and $\operatorname{Met}(T \widetilde{F})$ has the topology of $C^{l-1}$-uniform convergence on compact sets $(l \geq 1)$,

(3) if $\Phi: M \rightarrow M$ is an $\mathscr{F}$-preserving diffeomorphism and $\tilde{\Phi}$ denotes the corresponding automorphism of $P$ then $\widetilde{\Phi^{*}(\xi)}=(\widetilde{\Phi})^{*}(\tilde{\xi})$.

Proof. Take inspiration from [2, bottom of page 160 and top of page 161].

For each integer $k$, let $J_{\tilde{\mathscr{g}}}^{k}(P, \mathbf{R}) \rightarrow P$ denote the vector bundle of $k$-jets of smooth real valued functions on $P$ in the direction of $\widetilde{F}$, i.e., $J_{\widetilde{F}}^{k}(P, \mathbf{R})=$ $C^{\infty}(P, \mathbf{R}) / \sim$ where $C^{\infty}(P, \mathbf{R})$ denotes the space of smooth real-valued func- 
tions on $P$ and

$$
f \tilde{u}_{\tilde{u}} g \Leftrightarrow h(u)=0,\left.\quad h^{\prime}\right|_{T_{u} \tilde{\mathscr{F}}}=0, \ldots,\left.h^{(k)}\right|_{T_{u} \tilde{\mathscr{F}}=0} \text { where } h=f-g,
$$

and let $\operatorname{Met}\left(J_{\tilde{g}}^{k}(P, \mathbf{R})\right) \rightarrow P$ denote the bundle whose sections are smooth Riemannian metrics on $J_{\widetilde{F}}^{k}(P, \mathbf{R})$. The following proposition holds:

Proposition 4.2.2. There is a natural map from $\operatorname{Mex}(T \widetilde{\mathscr{F}})$ into $\operatorname{Met}\left(J_{\widetilde{\mathscr{F}}}^{k}(P, \mathbf{R})\right)$ sending $\tilde{\xi}$ to $\tilde{\xi}_{k}$ with the following properties:

(1) If $\Phi$ is an $\mathscr{F}$-preserving diffeomorphim of $M$ and $\tilde{\xi} \in \operatorname{Met}(T \widetilde{F})$ then $\left(\tilde{\Phi}^{*}(\tilde{\xi})\right)_{k}=(\tilde{\Phi})_{k}^{*}\left[\widetilde{\xi}_{k}\right]$.

(2) For any $l \geq k$ the map is continuous where $\operatorname{Met}(T \widetilde{F})$ has the topology of $C^{l}$-uniform convergence on compact sets and $\operatorname{Met}\left(J_{\widetilde{F}}^{k}(P, \mathbf{R})\right)$ has the topology of $C^{l-k}$-uniform convergence on compact sets.

Proof. Take inspiration from the proof of [2, Proposition 2.5, p. 162].

Let $\omega$ denote the smooth a-invariant Riemannian metric on $T \mathscr{F}$. By Proposition 4.2.1 $\omega$ yields a smooth Riemannian metric $\tilde{\omega}$ on $T \widetilde{F}$ which is both $G L(p)$ - and $\tilde{a}$-invariant, and which in turn, by Proposition 4.2.2, yields for each integer $k$ a smooth Riemannian metric $\tilde{\omega}_{k}$ on the vector bundle $J_{\widetilde{\mathcal{F}}}^{k}(P, \mathbf{R})$ which is both $G L(p)$ - and $\tilde{a}$-invariant.

We now see that the existence of a measurable Riemannian metric on $T \mathscr{F}$ which is a.e. invariant under the perturbed action $a^{\prime}$ implies the existence, for each integer $k$, of a measurable Riemannian metric $\tilde{\eta}_{k}^{\prime}$ on $J_{\tilde{\mathcal{F}}}^{k}(P, \mathbf{R})$ which is both $G L(p)$-invariant and a.e. $\tilde{a}^{\prime}$-invariant.

We first give a new construction of $J_{\widetilde{\widetilde{F}}}^{k}(P, \mathbf{R})$ as a balanced product: let $G\left(p+p^{2}\right)$ be the group of germs at $0 \in \mathbf{R}^{p+p^{2}}$ of local diffeomorphisms of $\mathbf{R}^{p+p^{2}}$ fixing 0 . For $k \geq 1$, let $G_{k}\left(p+p^{2}\right)$ be the normal subgroup consisting of diffeomorphisms that agree with the identity up to order $k$ and let $G L\left(p+p^{2}\right)^{(k)}$ be the quotient $G\left(p+p^{2}\right) / G_{k}\left(p+p^{2}\right)$. Let $J^{k}\left(\mathbb{R}^{p+p^{2}}, 0, \mathbf{R}\right)$ be the vector space of $k$-jets at 0 of smooth $\mathrm{R}$-valued functions on $\mathrm{R}^{p+p^{2}}$. If $\phi \in G L\left(p+p^{2}\right)^{(k)}$ and $f \in J^{k}\left(\mathbf{R}^{p+p^{2}}, 0, \mathbf{R}\right)$ then $(\phi, f) \mapsto f \circ \phi^{-1}$ defines a faithful left linear action of $G L\left(p+p^{2}\right)^{(k)}$ on $J^{k}\left(\mathbb{R}^{p+p^{2}}, 0, \mathbb{R}\right)$. Let $P_{\tilde{g}}^{(k)} \rightarrow P$ be the bundle of $k$-jets along the leaves of $\widetilde{F}$ of local diffeomorphisms of $P$ (an element of the fiber over $u \in P$ is a class of local diffeomorphisms of $P$ fixing $u$ which derivatives restricted to $\widetilde{D}_{u}=T_{u} \widetilde{F}$ agree up to order $k$ ): it is a principal $G L\left(p+p^{2}\right)^{(k)}$ fiber bundle and we have

$$
J_{\widetilde{F}}^{k}(P, \mathbf{R})=P_{\widetilde{F}}^{(k)} \times_{G L\left(p+p^{2}\right)(k)} J^{k}\left(\mathbf{R}^{p+p^{2}}, 0, \mathbf{R}\right) .
$$

Proposition 4.2.3.

(1) Let $\alpha^{(k)}$ be a cocycle corresponding to a measurable trivialization of $P_{\tilde{F}}^{(k)} \rightarrow P$ and the H-action on $P_{\tilde{F}}^{(k)}$ induced by $\tilde{a}^{\prime}$, and let $\alpha$ be the cocycle corresponding to $\tilde{a}^{\prime}$ and the associated trivialization of $P \rightarrow M$. 
As $\alpha$ is equivalent to a cocycle taking values in a compact subgroup of $G L(p), \alpha^{(k)}$ is also equivalent to a cocycle taking values in a compact subgroup of $G L\left(p+p^{2}\right)^{(k)}$ : thus, for each integer $k$, there exists a measurable Riemannian metric $\widetilde{\Upsilon}_{k}^{\prime}$ on $J_{\tilde{\sigma}}^{k}(P, \mathbf{R})$ which is a.e. $\tilde{a}^{\prime}$-invariant.

(2) If there exists such a measurable a.e. $\tilde{a}^{\prime}$-invariant Riemannian metric $\tilde{\Upsilon}_{k}^{\prime}$ on $J_{\tilde{g}}^{k}(P, \mathbf{R})$, then there exists a measurable Riemannian metric $\tilde{\eta}_{k}^{\prime}$ on $J_{\tilde{\mathscr{g}}}^{k}(P, \mathbf{R})$ which is both $G L(p)$-invariant and a.e. $\tilde{a}^{\prime}$-invariant.

Proof. (1) follows from [2, Proposition 4.7, p. 173] and (2) follows from [2, Corollary 4.13, p. 176].

4.3. The volume $\tilde{v^{\prime}}$ and the Sobolev spaces $L_{\tilde{\mathscr{F}}, \tilde{\eta}_{k}^{\prime}}^{s, k}(P, \mathbf{R})$. The $a^{\prime}$-invariant smooth volume $v^{\prime}$ on $M$ together with Haar measure $\mu$ on $G L(p)$ (which is unimodular) yields a smooth volume $\tilde{v^{\prime}}$ on $P$ which is both $G L(p)$ - and $\tilde{a}^{\prime}$-invariant.

Each $f \in C^{\infty}(P, \mathbf{R})$ defines a $k$-jet extension $j_{\widetilde{g}}^{k}(f) \in C^{\infty}\left(P, J_{\widetilde{F}}^{k}(P, \mathbf{R})\right)$. Let $\tilde{\eta}_{k}^{\prime}$ be the a.e. $\tilde{a}^{\prime}$-invariant measurable Riemannian metric on the vector bundle $J_{\tilde{g}}^{k}(P, \mathbf{R}) \rightarrow P$ obtained in $\S 4.2$ and set

$$
C^{\infty}(\boldsymbol{P}, \mathbf{R})_{\tilde{\mathscr{F}}, \tilde{\eta}_{k}^{\prime}}^{s, k}=\left\{f \in C^{\infty}(\boldsymbol{P}, \mathbf{R}) \mid\left\|j_{\widetilde{\mathcal{F}}}^{k}(f)\right\|_{\tilde{\eta}_{k}^{\prime}} \in L_{\tilde{v}^{\prime}}^{s}(P)\right\} .
$$

The set $C^{\infty}(P, \mathbf{R})_{\tilde{\mathscr{F}}, \tilde{\eta}_{k}^{\prime}}^{s, k}$ with the norm

$$
\|f\|_{\tilde{\mathscr{F}}, \tilde{\eta}_{k}^{\prime}}^{s, k}=\left(\int_{P}\left\|j_{\tilde{\mathscr{F}}}^{k}(f)\right\|_{\tilde{\eta}_{k}^{\prime}}^{s} d \tilde{v^{\prime}}\right)^{1 / s}
$$

is a normed linear vector space whose completion we denote by $L_{\widetilde{\mathscr{F}}, \widetilde{\eta}_{k}^{\prime}}^{s, k}(P, \mathbb{R})$. If the Riemannian metric $\tilde{\eta}_{k}^{\prime}$ is only measurable we might have $L_{\widetilde{\mathcal{F}}, \tilde{\eta}_{k}^{\prime}}^{s, k}(P, \mathbf{R})=0$. But if $\tilde{\eta}_{k}^{\prime}$ is smooth we clearly have $L_{\tilde{g}, \tilde{\eta}_{k}^{\prime}}^{s, k}(P, \mathbf{R}) \supset C_{c}^{\infty}(P, \mathbf{R})$, the smooth compactly supported functions on $P$. We are going to see that, provided $\tilde{\eta}_{k}^{\prime}$ satisfies some integrability conditions with respect to the smooth metric $\tilde{\omega}_{k}$, this inclusion also holds, together with a Sobolev-embedding type theorem. We first introduce the following definitions:

Definition 4.3.1. Let $V$ be a real vector space and let $\eta, \xi \in \operatorname{Inn}(V)$. Set $M(\eta / \xi)=\max \left\{\|x\|_{\eta} \mid\|x\|_{\xi}=1\right\}$. If $E \rightarrow P$ is a vector bundle and $\eta, \xi$ are measurable metrics on $E$, then let $M(\eta / \xi): \rightarrow \mathbf{R}$ be the measurable function defined by $M(\eta / \xi)(u)=M\left(\eta_{u} / \xi_{u}\right)$.

We know that there exists an atlas $\mathscr{A}=\left(h_{\alpha}\right)_{\alpha \in\{1, \ldots, i\}}$ on $M$ whose charts $h_{\alpha}: U_{\alpha} \rightarrow M$ trivialize $\mathscr{F}$. To this atlas corresponds the atlas

$$
\widetilde{\mathscr{A}}=\left({\widetilde{h_{\alpha}}}_{\alpha \in\{1, \ldots, i\}}\right.
$$

of $P$ whose charts $\widetilde{h_{\alpha}}: U_{\alpha} \times G L(p) \rightarrow P$ trivialize $\widetilde{\mathscr{F}}$. 
Definition 4.3.2. Let $f: P \rightarrow \mathbf{R}$ be a function on $P$. We will say that $f$ is $C^{r}$-for-almost-every-leaf-of- $\widetilde{F}$ if in each chart of the atlas $\widetilde{\mathscr{A}}$ and for almost every $x \in \pi_{2}\left(U_{\alpha}\right) \subset \mathbb{R}^{q}$ (where $q=d-p$ is the codimension of the foliation $F)$ the function $f \circ \tilde{h}_{\alpha}(., x,$.$) is C^{r}$.

We then have the following proposition:

Proposition 4.3.3.

(1) If $M\left(\tilde{\eta}_{k}^{\prime} / \tilde{\omega}_{k}\right) \in L_{\text {loc }}^{2}(P)$, then $C_{c}^{\infty}(P, \mathbf{R}) \subset L_{\widetilde{g}, \tilde{\eta}_{k}^{\prime}}^{2, k}(P, \mathbf{R})$.

(2) If $M\left(\tilde{\omega}_{k} / \tilde{\eta}_{k}^{\prime}\right) \in L_{\text {loc }}^{2}(P)$, then $L_{\tilde{\mathscr{F}}, \tilde{\eta}_{k}^{\prime}}^{2, k}(P, \mathbf{R}) \subset L_{\tilde{\mathscr{F}}, \tilde{\omega}_{k}, \text { loc }}^{1, k}(P, \mathbf{R})$.

(3) If $f \in L_{\tilde{\mathscr{F}}, \tilde{\omega}_{k}, \text { loc }}^{1, k}(P, \mathbf{R})$ and $k>r+\operatorname{dim}(\widetilde{\mathscr{F}})$, then $f$ is $C^{r}$-for-almostevery-leaf-of- $\widetilde{\mathscr{F}}$.

(4) Assume that $f: P \rightarrow \mathbf{R}$ is $C^{r}$-for-almost-every-leaf-of- $\widetilde{F}$. If $f$ is moreover $\tilde{a}^{\prime}$-invariant then $f \in C^{r}(P)$.

Proof. For (1) and (2) take inspiration from [2, Lemma 2.8, p. 164]. To prove (3) apply Fubini's Theorem and the classical Sobolev Embedding Theorem in the charts of the atlas $\widetilde{A}$, and to prove (4) use the $\tilde{a^{\prime}}$-invariance of $f$ together with the transversality of the $H$-action $\tilde{a}^{\prime}$ on $P$ to the foliation $\widetilde{F}$.

We now show that the integrability conditions of Proposition 4.3.3 (namely that $M\left(\tilde{\eta}_{k}^{\prime} / \tilde{\omega}_{k}\right)$ and $M\left(\tilde{\omega}_{k} / \tilde{\eta}_{k}^{\prime}\right)$ both belong to $\left.L_{\text {loc }}^{2}(P)\right)$ are realized provided that the perturbed action $a^{\prime}$ is $C^{\infty}$-close to $a$ :

Proposition 4.3.4. If $a^{\prime}$ is a sufficiently $C^{\infty}$-small perturbation of a then for each integer $k, M\left(\tilde{\eta}_{k}^{\prime} / \tilde{\omega}_{k}\right)$ and $M\left(\tilde{\omega}_{k} / \tilde{\eta}_{k}^{\prime}\right)$ both belong to $L_{\text {loc }}^{2}(P)$.

Proof. In order to insure that $M\left(\tilde{\eta}_{k}^{\prime} / \tilde{\omega}_{k}\right)$ belongs to $L_{\text {loc }}^{2}(P)$ we will examine the growth of this function along $\tilde{a}^{\prime}$-orbits and show that this can be converted into the desired integrability property by an application of Kazhdan's property. We first make the following remark: if $\tilde{\eta}_{k}^{\prime}$ and $\tilde{\omega}_{k}$ are both $G L(p)$-invariant, then $M\left(\tilde{\eta}_{k}^{\prime} / \widetilde{\omega}_{k}\right)$ can be considered as a function on $M$ so that in order to show that this function, considered as a function on $P$, belongs to $L_{\text {loc }}^{2}(P)$, it is enough to show that, considered as a function on $M$, it is in $L_{v^{\prime}}^{2}(M)$. Furthermore, as $\tilde{\eta}_{k}^{\prime}$ is $\tilde{a}^{\prime}$-invariant, then for each $h \in H$ and a.e. $m \in M$ we have

$$
\begin{aligned}
M\left(\tilde{\eta}_{k}^{\prime} / \tilde{\omega}_{k}\right)(h m) & =M\left(h^{*} \tilde{\eta}_{k}^{\prime} / h^{*} \tilde{\omega}_{k}\right)(m) \\
& \leq M\left(h^{*} \tilde{\eta}_{k}^{\prime} / \widetilde{\omega}_{k}\right)(m) M\left(\tilde{\omega}_{k} / h^{*} \widetilde{\omega}_{k}\right)(m) \\
& =M\left(\widetilde{\eta}_{k}^{\prime} / \widetilde{\omega}_{k}\right)(m) M\left(\widetilde{\omega}_{k} / h^{*} \tilde{\omega}_{k}\right)(m) .
\end{aligned}
$$

Thus the growth of $M\left(\tilde{\eta}_{k}^{\prime} / \tilde{\omega}_{k}\right)(m)$ along the $a^{\prime}$-orbit $H m$ is governed by the growth of $\left\|M\left(\widetilde{\omega}_{k} / h^{*} \widetilde{\omega}_{k}\right)\right\|_{\infty}$ (we recall that $\omega$ is smooth) along the same orbit. The following proposition is then clearly germane:

Proposition 4.3.5 [2, Theorem 8.1, p. 187]. Assume that $H$ is a Kazhdan group. Then there exist a compact subset $K_{0} \subset H$ and a constant $C_{0}>1$ (depending only on the group $H$ ) satisfying the following property: whenever $H$ acts ergod- 
ically on a probability space $(X, \mu)$ preserving $\mu$, any measurable $f: X \rightarrow \mathbb{R}$ which satisfies, for all $h \in K,|f(h x)| \leq C_{0}|f(x)|$ for a.e. $x \in X$, belongs to $L_{\mu}^{2}(X)$.

Thus by Proposition 4.3.5 to insure that $M\left(\tilde{\eta}_{k}^{\prime} / \tilde{\omega}_{k}\right) \in L_{v^{\prime}}^{2}(M)$ it is enough to insure that for all $h \in K_{0},\left\|M\left(\tilde{\omega}_{k} / h^{*} \widetilde{\omega}_{k}\right)\right\|_{\infty} \leq C_{0}$. But by (2) of Proposition 4.2.1 and (2) of Proposition 4.2.2 $\left\|M\left(\widetilde{\omega}_{k} / h^{*} \widetilde{\omega}_{k}\right)\right\|_{\infty}$ will be uniformly close to 1 for all $h \in K_{0}$ provided that $a^{\prime}$ is a $C^{k+1}$-small perturbation of $a$.

A similar argument holds for $\left\|M\left(\tilde{\omega}_{k} / \tilde{\eta}_{k}^{\prime}\right)\right\|_{\infty}$.

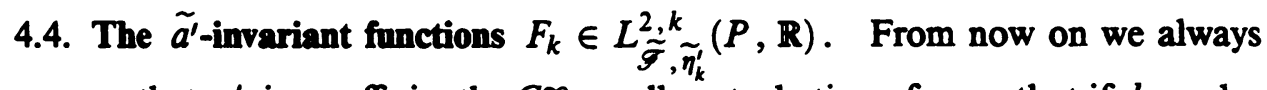
assume that $a^{\prime}$ is a sufficiently $C^{\infty}$-small perturbation of $a$ so that if $k$ and $r$ are integers such that $k>r+\operatorname{dim}(\widetilde{\mathscr{F}})$, we have $C_{c}^{\infty}(P, \mathbf{R}) \subset L_{\widetilde{\mathscr{F}}, \widetilde{\eta}_{k}^{\prime}}^{2, k}(P, \mathbb{R})$ and $L_{\tilde{\mathcal{F}}, \tilde{\eta}_{k}^{\prime}}^{2, k}(P, \mathbf{R}) \subset L_{\tilde{\mathscr{F}}, \tilde{\omega}_{k}, \text { loc }}^{1, k}(P, \mathbf{R}) \subset C^{r}(P)$. We can moreover assume that the Riemannian metric $\tilde{\eta}_{k}^{\prime}$ agrees with the standard metric on the naturally split trivial bundle $J_{\widetilde{F}}^{0}(P, \mathbf{R}) \subset J_{\tilde{F}}^{k}(P, \mathbf{R})$ so that $\|f\|_{\tilde{F}, \tilde{\eta}_{k}^{\prime}}^{2, k} \leq\|f\|_{2, \tilde{v^{\prime}}}$ for all $f \in C_{c}^{\infty}(P, \mathbf{R})$. By extending the identity on the subspace of smooth compactly supported functions we obtain canonical continuous injections $i_{k}$ : $L_{\widetilde{\widetilde{F}}, \tilde{\eta}_{k}^{\prime}}^{2, k}(P, \mathbf{R}) \hookrightarrow L_{\widetilde{v}^{\prime}}^{2}(P) \cap C^{r}(P)$ such that $\left\|i_{k}\right\|_{\text {op }} \leq 1$.

Proposition 4.4.1. For each integer $k$, the existence of a measurable Riemannian metric $\tilde{\eta}_{k}^{\prime}$ on the vector bundle $J_{\widetilde{g}}^{k}(P, \mathbf{R})$ which is both $G L(p)$-and $\widetilde{a}^{\prime}$-invariant and which moreover satisfies $M\left(\tilde{\eta}_{k}^{\prime} / \tilde{\omega}_{k}\right) \in L_{\text {loc }}^{2}(P)$ implies the existence of a nonzero $\tilde{a}^{\prime}$-invariant $F_{k} \in L_{\tilde{\mathscr{F}}, \tilde{\eta}_{k}^{\prime}}^{2, k}(P, \mathbf{R})$.

Proof. We first show that there exists a nonzero $\tilde{a}^{\prime}$-invariant $f \in L_{\tilde{v}^{\prime}}^{2}(P)$ : let $\Phi: M \times G L(p) \rightarrow P$ be a measurable trivialisation of $P$ such that the corresponding cocycle $\alpha: H \times M \rightarrow G L(p)$ satisfies $\alpha(H \times M) \subset K$ where $K \subset G L(p)$ is a compact subgroup (such a trivialization exists as $a^{\prime}$ leaves a measurable metric on $T \mathscr{F}$ a.e. invariant). It therefore suffices to see that there exists a nonzero $f \in L^{2}(M \times G L(p))$ which is $H$-invariant under the action $h .(m, g)=(h . m, \alpha(h, m) g)$. But clearly, if $\phi \in L^{2}(G L(p))$ is a nonzero left- $K$-invariant function, then $f(m, g)=\phi(g)$ is such a function.

Let $i_{k}: L_{\widetilde{g}, \tilde{\eta}_{k}^{\prime}}^{2, k}(P, \mathbf{R}) \rightarrow L_{\widetilde{v}^{\prime}}^{2}(P)$ denote the canonical inclusion. Since $\widetilde{\eta}_{k}^{\prime}$ and $\tilde{v}^{\prime}$ are $\tilde{a}^{\prime}$-invariant the $H$-action $\tilde{a}^{\prime}$ on $P$ induces unitary representations of $H$ on $L_{\widetilde{g}, \tilde{\eta}_{k}^{\prime}}^{2, k}(P, \mathbf{R})$ and $L_{\widetilde{v}^{\prime}}^{2}(P)$ respectively. Clearly $i_{k}(h . f)=h . i_{k}(f)$, i.e., $i_{k}$ is an intertwining operator between these two unitary representations. Let $\left(i_{k}\right)^{*}:\left(L_{\widetilde{v}^{\prime}}^{2}(P)\right)^{*} \rightarrow\left(L_{\widetilde{\mathcal{F}}, \tilde{\eta}_{k}^{\prime}}^{2, k}(P, \mathbb{R})\right)^{*}$ denote as usual the dual map of $i_{k}$, and let $d_{k}:\left(L_{\widetilde{\mathcal{F}}, \widetilde{\eta}_{k}^{\prime}}^{2, k}(P, \mathbf{R})\right)^{*} \rightarrow L_{\widetilde{\mathcal{F}}, \tilde{\eta}_{k}^{\prime}}^{2, k}(P, \mathbb{R})$ and $d:\left(L_{\widetilde{v}^{\prime}}^{2}(P)\right)^{*} \rightarrow L_{\widetilde{v}^{\prime}}^{2}(P)$ denote respectively the canonical maps identifying $L_{\widetilde{F}, \widetilde{\eta}_{k}^{\prime}}^{2, k}(P, R)$ and $L_{\widetilde{v}^{\prime}}^{2}(P)$ with their duals. 


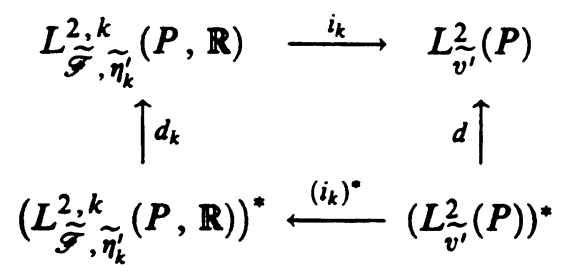

The map $\Psi_{k}=d_{k} \circ i_{k}^{*} \circ d^{-1}$ also intertwines the two unitary representations of $H$ so that if $f \in L_{\widetilde{v}^{\prime}}^{2}(P)$ is $H$-invariant, $\Psi_{k}(f) \in L_{\widetilde{\mathscr{F}}, \tilde{\eta}_{k}^{\prime}}^{2, k}(P, \mathbf{R})$ will also be $H$ invariant. Since $i_{k}\left(L_{\widetilde{F}, \tilde{\eta}_{k}^{\prime}}^{2, k}(P, \mathbf{R})\right)$ contains $C_{c}^{\infty}(P, \mathbf{R})$ (by (1) of Proposition 4.3.3) it is dense in $L_{\widetilde{v}^{\prime}}^{2}(P)$ and thus $\left(i_{k}\right)^{*}$ is injective. Thus the function $F_{k}=\Psi_{k}(f) \in L_{\tilde{\widetilde{\sigma}}, \widetilde{\eta}_{k}^{\prime}}^{2, k}(P, \mathbf{R})$ (where $f \in L_{\widetilde{v}^{\prime}}^{2}(P)$ is the nonzero $H$-invariant function obtained before) is nonzero and $H$-invariant.

4.5. Proof of the Main Theorem. Let $a^{\prime}$ be a sufficiently $C^{\infty}$-small perturbation of $a$ and fix an integer $k$. In $\$ 4.2$ we have proved the existence of a measurable Riemannian metric $\tilde{\eta}_{k}^{\prime}$ on the vector bundle $J_{\tilde{F}}^{k}(P, \mathbf{R}) \rightarrow P$ which is both $G L(p)$ - and $\tilde{a}^{\prime}$-invariant, and the existence of a smooth Riemannian metric $\widetilde{\omega}_{k}$ on the same vector bundle which is both $G L(p)$ - and $\tilde{a}$-invariant. By Proposition 4.3.4 the metric $\widetilde{\eta}_{k}^{\prime}$ necessarily satisfies the integrability conditions (1) and (2) of Proposition 4.3.3 (with respect to the smooth metric $\widetilde{\omega}_{k}$ ).

From now on we fix such a perturbation $a^{\prime}$ and two integers $k$ and $r$ such that $r \geq 1$ and $k>r+\operatorname{dim}(\widetilde{F})$.

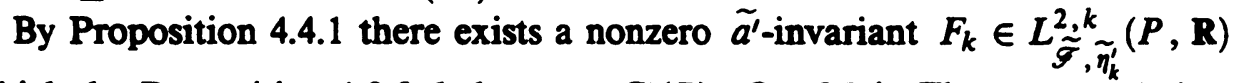
which, by Proposition 4.3.3, belongs to $C^{r}(P)$. Our Main Theorem 4.1.1 thus clearly follows from the following proposition:

Proposition 4.5.1. The existence of such an $F_{k} \in L_{\tilde{\mathcal{F}}, \tilde{\eta}_{k}^{\prime}}^{2, k}(P, \mathbb{R}) \cap C^{r}(P)$ implies the existence of an $a^{\prime}$-invariant $C^{r}$-Riemannian metric on $T \mathscr{F}$.

We recall the latter is equivalent to the existence of a compact subgroup $K \subset G L(p)$ and an $a^{\prime}$-invariant $C^{r}$ cross-section $\sigma^{\prime}: M \rightarrow P / K$ of the natural projection $P / K \rightarrow M$.

Lemma 4.5.2. Assume that $f \in C^{r}(P) \cap L_{\widetilde{v}^{\prime}}^{2}(P)$ (respectively $f \in C^{r}(P) \cap L_{\widetilde{v}^{\prime}}^{1}(P)$ )

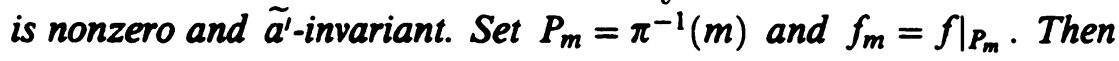

(1) $f_{m} \in L^{2}\left(P_{m}\right)$ (respectively $L^{1}\left(P_{m}\right)$ ) for all $m \in M$.

(2) Let $\Pi$ denote the left regular representation of $G L(p)$ on $L^{2}(G L(p))$ (respectively on $L^{1}(G L(p))$ ) and set $W=\left\{m \in M \mid f_{m} \neq 0\right\}$. Then $W$ is an open $H$-invariant conull set and there exists $\lambda \in C^{r}(G L(p)) \cap$ $L^{2}(G L(p))$ (respectively $C^{r}(G L(p)) \cap L^{1}(G L(p))$ ) such that in any trivialization of $P$ and for all $m \in W$ we have $f_{m} \in \Pi(G L(p))(\lambda)$.

Proof. We will prove Lemma 4.5.2 for $f \in L_{v^{\prime}}^{2}(P)$, the proof for $f \in L_{v^{\prime}}^{1}(P)$ being identical. Fix $\Phi: M \times G L(p) \rightarrow P$ a measurable trivialization of $P$ and 
let $\alpha: H \times M \rightarrow G L(p)$ be the cocycle corresponding to the $H$-action $\tilde{a}^{\prime}$ on $P$ and the trivialization $\Phi$. We can then look upon $f$ as a measurable function on $M \times G L(p)$, and $f_{m}$ as a $C^{r}$-function on $G L(p)$. As $f$ is $H$-invariant, for all $m \in M, g \in G L(p)$ and $h \in H$ we have

$$
f(\Phi(m, g))=f(h \Phi(m, g))=f(\Phi(h m, \alpha(h, m) g))
$$

and thus

$$
f(\Phi(h m, .))=\Pi(\alpha(h, m))[f(\Phi(m, .))] \text {. }
$$

Set $W_{1}=\left\{m \in M \mid f_{m} \in L^{2}\left(P_{m}\right)\right\}$ and let $\beta: W_{1} \rightarrow L^{2}(G L(p))$ be the map $m \mapsto f(\Phi(m,)$.$) . Let q: L^{2}(G L(p)) \rightarrow L^{2}(G L(p)) / G L(p)$ be the canonical projection associated to the left regular representation of $G L(p)$ on $L^{2}(G L(p))$ and define $\bar{f}: M \rightarrow L^{2}(G L(p)) / G L(p)$ by $\bar{f}=q \circ \beta$. By Fubini $W_{1}$ is conull. By $(*) W_{1}$ is $H$-invariant and for all $h \in H$ we have $\overline{f(h m)}=$ $\overline{f(m)}$ a.e. in $W_{1}$. As the $H$-action $a^{\prime}$ on $M$ is assumed to be ergodic, by [1, Proposition 2.1 .11$, p. 11] the function is constant a.e. on $W_{1}$ provided that the space $L^{2}(G L(p)) / G L(p)$ is countably separated:

Claim 1 . The space $L^{2}(G L(p)) / G L(p)$ is countably separated.

Proof. By [1, Proposition 2.1.14, p. 12], Claim 1 is equivalent to saying that each $G L(p)$-orbit in $L^{2}(G L(p))$ is locally closed. In fact, for any lesc group $G$, any $G$-orbit in $L^{2}(G)$ is closed.

Thus there exists $\lambda \in L^{2}(G L(p))$ and $W_{2} \subset W_{1}$ conull and $H$-invariant such that

$$
f(\Phi(m, .)) \in \Pi(G L(p)) \lambda \quad \text { for all } m \in W_{2} .
$$

This implies that $\lambda \in C^{r}(G L(p))$ and $\lambda \neq 0$.

Claim 2. $W_{1}=M$ (this proves (1)).

Proof. Fix $m_{0} \in M$. As $W_{2}$ is conull there exists a sequence $\left(m_{n}\right)_{n \in \mathbb{N}}$ of points of $W_{2}$ such that $m_{n} \underset{n \rightarrow \infty}{\longrightarrow} m_{0}$. Fix some continuous trivialization of $P$ centered at $m_{0}$, say $\Psi: U\left(m_{0}\right) \times G L(p) \rightarrow \pi^{-1}\left(U\left(m_{0}\right)\right)$. Then by definition of $W_{2}$ there exists a sequence $\left(g_{n}\right)_{n \in \mathbb{N}}$ of elements of $G L(p)$ such that

$$
f\left(\Psi\left(m_{n}, .\right)\right)=\Pi\left(g_{n}\right) \lambda=f_{n} .
$$

By continuity of $\Psi$ and $f, \varliminf_{n \rightarrow \infty}\left|f_{n}\right|=\left|f\left(\Psi\left(m_{0},.\right)\right)\right|$, and by Fatou's Lemma,

$$
\begin{aligned}
\int_{G L(p)} \varliminf_{n \rightarrow \infty}\left|f_{n}\right|^{2} d g & =\int_{G L(p)}\left|f\left(\Psi\left(m_{0}, .\right)\right)\right|^{2} d g \\
& \leq \varliminf_{n \rightarrow \infty} \int_{G L(p)}\left|f_{n}\right|^{2} d g \leq \varliminf_{n \rightarrow \infty} \int_{G L(p)}\left|\lambda\left(g_{n} g\right)\right|^{2} d g=\|\lambda\|_{2}^{2}
\end{aligned}
$$

and thus $f_{m_{0}} \in L^{2}\left(P_{m_{0}}\right)$, i.e., $m_{0} \in W_{1}$.

Claim 3. Set $W=\left\{m \in M \mid f_{m} \neq 0\right\}$. Then $W=W_{2}$. (This proves (2).)

Proof. Clearly $W_{2} \subset W$ so we just have to show that $W \subset W_{2}$, i.e., that if $m_{0} \in W_{2}^{c}$ then $f_{m_{0}}=0$. Fix $m_{0} \in W_{2}^{c}$. As $W_{2}$ is conull there exists a sequence $\left(m_{n}\right)_{n \in \mathrm{N}}$ of points of $W_{2}$ such that $m_{n} \underset{n \rightarrow \infty}{\longrightarrow} m_{0}$. As in the proof of Claim 2 , fix some continuous trivialization centered at $m_{0}, \Psi: U\left(m_{0}\right) \times G L(p) \rightarrow$ 
$\pi^{-1}\left(U\left(m_{0}\right)\right)$. By definition of $W_{2}$ there exists a sequence $\left(g_{n}\right)_{n \in \mathbb{N}}$ of elements of $G L(p)$ such that

$$
f\left(\Psi\left(m_{n}, .\right)\right)=\Pi\left(g_{n}\right) \lambda=f_{n} .
$$

Assume that $\left(g_{n}\right)$ has a convergent subsequence $g_{n_{k}} \underset{k \rightarrow \infty}{\longrightarrow} g_{0}$. Then $\Pi\left(g_{n_{k}}\right)$ $\underset{k \rightarrow \infty}{\longrightarrow} \Pi\left(g_{0}\right) \lambda$ pointwise in $G L(p)$ and by continuity of $\stackrel{\infty}{\Psi}$ and $f$,

$$
\Pi\left(g_{n_{k}}\right) \lambda=f\left(\Psi\left(m_{n_{k}}, .\right)\right) \underset{k \rightarrow \infty}{\longrightarrow} f\left(\Psi\left(m_{0}, .\right)\right) \quad \text { pointwise in } G L(p),
$$

and thus $f\left(\Psi\left(m_{0},.\right)\right)=\Pi\left(g_{0}\right) \lambda$, which contradicts the assumption that $m_{0} \in$ $W_{2}^{c}$. We thus necessarily have $g_{n} \underset{n \rightarrow \infty}{\longrightarrow} \infty$ so that

$$
\Pi\left(g_{n}\right)(\lambda)=f\left(\Psi\left(m_{n}, .\right)\right) \stackrel{L_{l o c}^{2}(G L(p))}{n \rightarrow \infty} 0 .
$$

It is therefore enough to see that

$$
f\left(\Psi\left(m_{n}, .\right)\right) \underset{n \rightarrow \infty}{\stackrel{L_{0 c}^{2}(G L(p))}{\longrightarrow}} f\left(\Psi\left(m_{0}, .\right)\right) .
$$

Fix $K \subset G L(p)$ a compact. By continuity of $\Psi$,

$$
f\left(\Psi\left(m_{n}, .\right)\right) \underset{n \rightarrow \infty}{\longrightarrow} f\left(\Psi\left(m_{0}, .\right)\right)
$$

pointwise in $G L(p)$. Without loss of generality we can assume that $U\left(m_{0}\right)$ is relatively compact and thus that for all integers $N$ and all $g \in K$

$$
\left|f\left(\Psi\left(m_{n}, g\right)\right)-f\left(\Psi\left(m_{0}, g\right)\right)\right|^{2} \leq C .
$$

As Haar measure is finite on compact sets $C \in L_{K}^{2}(G L(p))$, so that we are done by Lebesgue's Dominated Convergence Theorem.

Lemma 4.5.3. Let $F_{k} \in L_{\widetilde{F}, \widetilde{\eta}_{k}^{\prime}}^{2, k}(P, \mathbf{R}) \subset C^{r}(P) \cap L_{\widetilde{v}^{\prime}}^{2}(P)$ be the nonzero $\tilde{a^{\prime}}$ invariant function on $P$ obtained at the beginning of $\S 4.5$, and let $W=\{m \in$ $\left.M \mid\left(F_{k}\right)_{m} \neq 0\right\}$ be the $a^{\prime}$-invariant open conull subset of $M$ introduced in (2) of Lemma 4.5.2. Fix $m_{0} \in W^{c}$. Then there exists a nonzero $\tilde{a}^{\prime}$-invariant function $f^{m_{0}} \in L_{\widetilde{F}, \tilde{\eta}_{k}^{\prime}}^{2, k}(P, \mathbf{R})$ such that $\left(f^{m_{0}}\right)_{m_{0}} \neq 0$.

Proof. As the $H$-action $a^{\prime}$ on $M$ is transverse to the foliation $\mathscr{F}$, there exist vectors $H_{1}, \ldots, H_{q} \in \mathfrak{h}$ (where $\mathfrak{h}$ denotes the Lie algebra of $H$ and $q=d-p$ is the codimension of $\mathscr{F})$ such that $T_{m_{0}} M=T_{m_{0}} \mathscr{F} \oplus\left\langle k^{H_{1}}\left(m_{0}\right), \ldots, k^{H_{q}}\left(m_{0}\right)\right\rangle$ (where the second factor denotes the subspace spanned at $m_{0}$ by the the Killing fields $\left.k^{H_{i}}\right)$. Let $\phi: V(0) \rightarrow V_{L_{m_{0}}}\left(m_{0}\right)$ be a local chart of the leaf $L_{m_{0}}$ centered at $m_{0}$ (where $V(0) \subset \mathbb{R}^{p}$ is an open convex relatively compact neighbourhood of the origin, so that $V_{L_{m_{0}}}\left(m_{0}\right) \subset L_{m_{0}}$ is an open connected relatively compact neighbourhood of $m_{0}$ in the leaf $\left.L_{m_{0}}\right)$, fix $\epsilon>0$ and define the map $\Phi$ : $V(0) \times(-\epsilon, \epsilon)^{q} \rightarrow M$ by

$$
\Phi\left(l, t_{1}, \ldots, t_{q}\right)=\exp \left(\sum_{i=1}^{i=q} t_{i} H^{i}\right) . \phi(l) .
$$

For each $(l, 0) \in V(0) \times(-\epsilon, \epsilon)^{q}$ the map $\Phi$ is a local diffeomorphism at $(l, 0)$ so that by picking $\epsilon$ small enough we can assume that $\Phi$ is a diffeomorphism:

$$
\Phi: V(0) \times(-\epsilon, \epsilon)^{q} \rightarrow U\left(m_{0}\right)
$$


where $U\left(m_{0}\right) \subset M$ is an open connected relatively compact neighbourhood of $m_{0} \in W^{c}$. As the $H$-action $a^{\prime}$ on $M$ permutes the leaves of the foliation $\mathscr{F}$ this diffeomorphism is moreover a local trivialisation of $\mathscr{F}$ which, by construction, is centered at $m_{0}$ and satisfies

$$
\Phi(V(0) \times\{0\})=\phi(V(0))=V_{L_{m_{0}}}\left(m_{0}\right) \subset L_{m_{0}} .
$$

We will thus refer to the coordinates $l$ and $t$ on $U\left(m_{0}\right)$ as, respectively, the leafwise and transverse coordinates.

As the map $\Phi$ is a local trivialisation of $\mathscr{F}$, it maps the canonical frame $\left(e_{1}, \ldots, e_{p}\right)$ at $(l, t) \in V(0) \times(-\epsilon, \epsilon)^{q} \subset \mathbb{R}^{p} \times \mathbf{R}^{q}$ onto a frame of $\mathscr{F}$ at $\Phi(l, t) \in U\left(m_{0}\right)$ which we denote $u(l, t) \in P$. Set $u(0,0)=u_{0}$ and let $\pi: P \rightarrow M$ be the natural projection. By construction $\pi\left(u_{0}\right)=m_{0}$ and $\pi^{-1}\left(U\left(m_{0}\right)\right)$ is an open neighbourhood of $u_{0}$. We can naturally define a diffeomorphism

$$
\tilde{\Phi}: V(0) \times(-\epsilon, \epsilon)^{q} \times G L(p) \rightarrow \pi^{-1}\left(U\left(m_{0}\right)\right)
$$

by the formula

$$
\tilde{\Phi}(l, t, g)=u(l, t) \cdot g
$$

which is both a local trivialization of the $G L(p)$-bundle $\pi^{-1}\left(U\left(m_{0}\right)\right) \rightarrow U\left(m_{0}\right)$ and a local trivialization of the foliation $\widetilde{F}$ on $P$ centered at $u_{0}$, which satisfies

$$
\tilde{\Phi}(V(0) \times\{0\} \times G L(p)) \subset \tilde{L}_{u_{0}} .
$$

The coordinates $(l, t, g)$ on $U\left(u_{0}\right)$ will be respectively referred to as the leafwise, transverse and group coordinates (note that leafwise is with respect to the foliation $\mathscr{F}$ and not $\widetilde{F}$ ).

As $W \subset M$ is $H$-invariant and, by hypothesis, $m_{0} \in W^{c}$, necessarily $\Phi\left(\{0\} \times \mathbb{R}^{q}\right) \subset W^{c}$ and $\Phi^{-1}\left(W \cap U\left(m_{0}\right)\right) \subset V(0) \times(-\epsilon, \epsilon)^{q}$ is invariant under small transverse translations (by this we mean that if $(l, t) \in V(0) \times$ $(-\epsilon, \epsilon)^{q}, t_{0} \in(-\epsilon, \epsilon)^{q}$, and $\Phi(l, t) \in W$, then $\left.\Phi\left(l, t_{0}\right) \in W\right)$. Therefore $W^{c} \cap V_{L_{m_{0}}}\left(m_{0}\right) \subset L_{m_{0}}$ must also have measure zero in $V\left(m_{0}\right) \subset L_{m_{0}}$ (otherwise $W^{c} \subset M$ would contain a subset of strictly positive measure). We can therefore assert that there exists a sequence $\left(m_{j}\right)_{j \in \mathrm{N}}$ such that

$$
m_{j} \in W \cap V_{L_{m_{0}}}\left(m_{0}\right) \subset L_{m_{0}} \text { and } m_{j} \underset{j \rightarrow \infty}{\longrightarrow} m_{0} .
$$

By (2) of Lemma 4.5.2 there exist a nonzero $\lambda \in C^{r}(G L(p)) \cap L^{2}(G L(p))$ and a sequence $\left(g_{j}\right)_{j \in \mathrm{N}}$ of elements of $G L(p)$ such that $\left(F_{k}\right)_{m_{j}}=\Pi\left(g_{j}\right) \lambda$ where $\left(F_{k}\right)_{m_{j}}(g)=F_{k} \circ \tilde{\Phi}\left(\Phi^{-1}\left(m_{j}\right), g\right)$. Set $u_{j}=\tilde{\Phi}\left(\Phi^{-1}\left(m_{j}\right), e\right) \in \tilde{L}_{u_{0}}$. By construction $\pi\left(u_{j}\right)=m_{j} \in L_{m_{0}}$. By continuity of $\Phi$ and $\tilde{\Phi}$, we have $u_{j}=\tilde{\Phi}\left(\Phi^{-1}\left(m_{j}\right), e\right) \underset{j \rightarrow \infty}{\longrightarrow} \tilde{\Phi}\left(\Phi^{-1}\left(m_{0}\right), e\right)=\tilde{\Phi}(0,0, e)=u_{0}$ and as $\lambda \neq 0$ there exists $g_{0} \in G L(p)$ such that $\lambda\left(g_{0}\right) \neq 0$. Set

$$
f_{j}=\Pi_{R}\left(g_{j} g_{0}\right)\left(F_{k}\right)
$$

where $\Pi_{R}$ denotes the right regular representation of $G L(p)$ on $L_{\widetilde{\mathscr{F}}, \tilde{\eta}_{k}^{\prime}}^{2, k}(P, \mathbb{R})$ (which is unitary as both the volume $\tilde{v}^{\prime}$ and the metric $\tilde{\eta}_{k}^{\prime}$ are $G L(p)$-invariant). 
Thus $f_{j} \in L_{\widetilde{\mathcal{F}}, \widetilde{\eta}_{k}^{\prime}}^{2, k}(P, \mathbf{R}),\left\|f_{j}\right\|_{\widetilde{\mathscr{F}}, \tilde{\eta}_{k}^{\prime}}^{2, k}=\left\|F_{k}\right\|_{\widetilde{\mathcal{F}}, \widetilde{\eta}_{k}^{\prime}}^{2, k}$, and as $H$ acts on the left of $P$ and $F_{k}$ is $\tilde{a}^{\prime}$-invariant (and nonzero), $f_{j}$ is also $\tilde{a}^{\prime}$-invariant (and nonzero): in order to prove Lemma 4.5.3 it is therefore enough to show that there exists an integer $j_{0}$ such that $f_{j_{0}}\left(u_{0}\right) \neq 0$ (we are then done by setting $f^{m_{0}}=f_{j_{0}}$ ).

Claim. There exists an integer $j_{0}$ such that $f_{j_{0}}\left(u_{0}\right) \neq 0$.

We prove this claim by contradiction: assume that for all $j \in \mathbb{N}$ we have $f_{j}\left(u_{0}\right)=0$. We will use the following fact:

$$
f_{j}\left(u_{j}\right)=f_{j}\left(\tilde{\Phi}\left(\Phi^{-1}\left(m_{j}\right), e\right)\right)=\left[\Pi_{R}\left(g_{j} g_{0}\right) \Pi\left(g_{j}\right) \lambda\right](e)=\lambda\left(g_{j}^{-1} e g_{j} g_{0}\right)=\lambda\left(g_{0}\right) .
$$

Let $V(e) \subset G L(p) \subset \mathbf{R}^{p^{2}}$ be an open convex relatively compact neighbourhood of the identity and set $U\left(u_{0}\right)=\widetilde{\Phi}\left(V(0) \times(-\epsilon, \epsilon)^{q} \times V(e)\right)$. Clearly $U\left(u_{0}\right)$ is an open connected relatively compact neighbourhood of $u_{0} \in P$ and by passing to a subsequence we can assume that the $u_{j}$ lie in $U\left(u_{0}\right)$. For each integer $j$ define a $C^{r}$-function

$$
G_{j}=f_{j} \circ \tilde{\Phi}: V(0) \times(-\epsilon, \epsilon)^{q} \times V(e) \rightarrow \mathbf{R}
$$

to which we can apply the Mean Value Theorem $\left(V(0) \times(-\epsilon, \epsilon)^{q} \times V(e) \subset\right.$ $\mathbf{R}^{p} \times \mathbf{R}^{q} \times \mathbf{R}^{p^{2}}$ being convex and open) between the points $\tilde{\Phi}^{-1}\left(u_{j}\right)$ and 0 : there exists $c_{j} \in\left[0, \tilde{\Phi}^{-1}\left(u_{j}\right)\right] \subset \mathbf{R}^{p} \times \mathbf{R}^{q} \times \mathbf{R}^{p^{2}}$ such that

$$
\begin{aligned}
d G_{j}\left(c_{j}\right)\left(\tilde{\Phi}^{-1}\left(u_{j}\right)-0\right) & =G_{j}\left(\tilde{\Phi}^{-1}\left(u_{j}\right)\right)-G_{j}(0)=f_{j}\left(u_{j}\right)-f_{j}\left(u_{0}\right) \\
& =f_{j}\left(u_{j}\right)=\lambda\left(g_{0}\right) .
\end{aligned}
$$

As $c_{j} \underset{j \rightarrow \infty}{\longrightarrow} 0$ (as $c_{j} \in\left[0, \tilde{\Phi}^{-1}\left(u_{j}\right)\right]$ ), and $\tilde{\Phi}^{-1}\left(u_{j}\right) \underset{j \rightarrow \infty}{\longrightarrow} 0$, we have

$$
\left\|d G_{j}\left(c_{j}\right)\right\| \geq \frac{\left|\lambda\left(g_{0}\right)\right|}{\left\|\tilde{\Phi}-1\left(u_{j}\right)\right\|} \underset{j \rightarrow \infty}{\longrightarrow} \infty
$$

where $\left\|d G_{j}\left(c_{j}\right)\right\|$ denotes the norm of the derivative $d G_{j}\left(c_{j}\right): \mathbf{R}^{p} \times \mathbf{R}^{q} \times \mathbf{R}^{p^{2}} \rightarrow$ $\mathbf{R}$. We moreover point out that as $u_{j} \in \tilde{L}_{u_{0}}$, by construction of $\tilde{\Phi}$ both $\tilde{\Phi}^{-1}\left(u_{j}\right)$ and $c_{j}$ lie in $V(0) \times\{0\} \times V(e)$ so that if $\bar{G}_{j}$ denotes the restriction of $G_{j}$ to $V(0) \times\{0\} \times V(e) \subset V(0) \times(-\epsilon, \epsilon)^{q} \times V(e)$ then

$$
\left\|d \bar{G}_{j}\left(c_{j}\right)\right\| \underset{j \rightarrow \infty}{\longrightarrow} \infty
$$

where $\left\|d \bar{G}_{j}\left(c_{j}\right)\right\|$ denotes this time the norm of the derivative $d \bar{G}_{j}\left(c_{j}\right): \mathbf{R}^{p} \times$ $\mathbf{R}^{p^{2}} \rightarrow \mathbf{R}$.

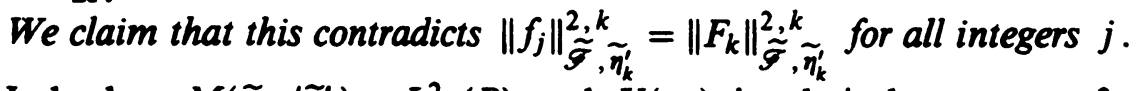

Indeed as $M\left(\tilde{\omega}_{k} / \tilde{\eta}_{k}^{\prime}\right) \in L_{\text {loc }}^{2}(P)$ and $U\left(u_{0}\right)$ is relatively compact, for each integer $j$ we have on one hand:

$$
\begin{aligned}
\int_{U\left(u_{0}\right)}\left\|j_{\tilde{\mathscr{F}}}^{k}\left(f_{j}\right)\right\|_{\tilde{\omega}_{k}} d \tilde{v}^{\prime} & \leq \int_{U\left(u_{0}\right)}\left\|j_{\tilde{\mathscr{F}}}^{k}\left(f_{j}\right)\right\|_{\tilde{\eta}_{k}^{\prime}} M\left(\tilde{\omega}_{k} / \tilde{\eta}_{k}^{\prime}\right) d \tilde{v}^{\prime} \\
& \leq\left(\int_{U\left(u_{0}\right)}\left\|j_{\tilde{\mathcal{F}}}^{k}\left(f_{j}\right)\right\|_{\tilde{\eta}_{k}^{\prime}}^{2} d \tilde{v^{\prime}}\right)^{1 / 2}\left(\int_{U\left(u_{0}\right)} M^{2}\left(\tilde{\omega}_{k} / \tilde{\eta}_{k}^{\prime}\right) d \tilde{v^{\prime}}\right)^{1 / 2} \\
& \leq\left\|f_{j}\right\|_{\tilde{\mathscr{F}}, \tilde{\eta_{k}^{\prime}}}^{2, k} C_{1}=\left\|F_{k}\right\|_{\tilde{\widetilde{F}}, \eta_{k}^{\prime}}^{2, k} C_{1} \leq C_{2}
\end{aligned}
$$


for some constants $C_{1}, C_{2}>0$, so that the quantities $\int_{U\left(u_{0}\right)}\left\|j_{\widetilde{\mathcal{g}}}^{k}\left(f_{j}\right)\right\|_{\tilde{\omega}_{k}} d \tilde{v^{\prime}}$ are uniformly bounded. On the other hand, by construction of $\widetilde{\boldsymbol{\Phi}}$, the formula of change of variables, the relative compactness of $U\left(u_{0}\right) \subset P$, Fubini's Theorem and the $\tilde{a}^{\prime}$-invariance of the functions $f_{j}$ (which implies in the chart $\widetilde{\Phi}$ the invariance of $G_{j}$ by transverse translations), there exist constants $C_{3}>0$ and $C_{4}>0$ such that

$$
\begin{aligned}
& \int_{U\left(u_{0}\right)}\left\|j_{\tilde{\mathcal{F}}}^{k}\left(f_{j}\right)\right\|_{\tilde{\omega}_{k}} d \tilde{v^{\prime}} \\
& =\int_{V(0) \times(-\epsilon, \epsilon) \times V(e)}\left\|j_{\widetilde{F}_{0}}^{k}\left(G_{j}\right)\right\|_{\tilde{\Phi}^{*}\left(\tilde{\omega}_{k}\right)} \frac{d\left[\tilde{\Phi}^{*}\left(\tilde{v}^{\prime}\right)\right]}{d l \times d t \times d g} d l \times d t \times d g \\
& \geq C_{3} \int_{V(0) \times(-\epsilon, \epsilon) \times \times V(e)}\left\|j_{\widetilde{g}_{0}}^{k}\left(G_{j}\right)\right\|_{0}(l, t, g) d l \times d t \times d g \\
& =(2 \epsilon)^{q} C_{3} \int_{V(0) \times V(e)}\left\|j_{\mathscr{G}_{0}}^{k}\left(\bar{G}_{j}\right)\right\|_{0}(l, g) d l \times d g \geq C_{4}\left\|\bar{G}_{j}\right\|_{1, k}^{V(0) \times V(e)}
\end{aligned}
$$

where $\widetilde{F_{0}}$ denotes the canonical foliation on $\mathbf{R}^{p} \times \mathbf{R}^{q} \times \mathbf{R}^{p^{2}}$ obtained by translating $\mathbf{R}^{p} \times\{0\} \times \mathbf{R}^{p^{2}}, j_{\widetilde{\mathscr{J}}_{0}}^{k}\left(G_{j}\right)$ denotes the $k$-jet of the mip $G_{j}$ in the direction of $\widetilde{\mathscr{F}_{0}},\|\|_{0}$ in $\left\|j_{\widetilde{\mathscr{F}}_{0}}^{k}\left(G_{j}\right)\right\|_{0}$ denotes the canonical norm on the vector bundle of the $k$-jets of functions on $\mathbf{R}^{p} \times \mathbf{R}^{q} \times \mathbf{R}^{p^{2}}$ in the direction of $\widetilde{F_{0}}, \mathscr{F}_{0}$ denotes the canonical foliation on $\mathbf{R}^{p} \times \mathbf{R}^{p^{2}}$ obtained by translating $\mathbf{R}^{p} \times\{0\}$, $j_{\mathscr{S}_{0}}^{k}\left(\bar{G}_{j}\right)$ denotes the $k$-jet of the map $\bar{G}_{j}$ in the direction of $\mathscr{F}_{0}$, and \|\|$_{0}$ in $\left\|j_{\mathscr{G}_{0}}^{k}\left(\bar{G}_{j}\right)\right\|_{0}$ denotes the canonical norm on the vector bundle of the $k$-jets of functions on $\mathrm{R}^{p} \times \mathrm{R}^{p^{2}}$ in the direction of $\mathscr{S}_{0}$. Thus the $\left\|\bar{G}_{j}\right\|_{1, k}^{V(0) \times V(e)}$ are uniformly bounded. We get the desired contradiction by applying the Sobolev Embedding Theorem: since we assumed $r \geq 1$ we have the following bounded injection:

$$
L^{1, k}(V(0) \times V(e)) \hookrightarrow B C^{1}(V(0) \times V(e)) .
$$

Therefore the $\left\|d \bar{G}_{j}\left(c_{j}\right)\right\|$ should remain bounded, which contradicts $(*)$.

Lemma 4.5.4. There exists an $\tilde{a}^{\prime}$-invariant $h \in C^{r}(P, \mathbf{R}) \cap L_{\tilde{v}^{\prime}}^{1}(P)$ such that $h_{m} \neq 0$ for all $m \in M$.

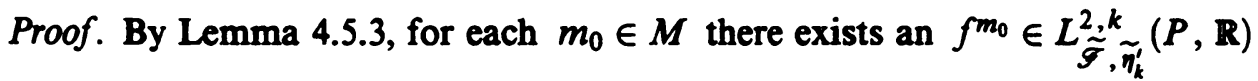
which is $\tilde{a}^{\prime}$-invariant and satisfies $\left(f^{m_{0}}\right)_{m_{0}} \neq 0$. As $f^{m_{0}} \in L_{\tilde{\mathscr{g}}, \tilde{\eta}_{k}^{\prime}}^{2, k}(P, \mathbf{R}) \subset$ $C^{r}(P, \mathbf{R})$, necessarily $\left(f^{m_{0}}\right)_{m} \neq 0$ for all $m \in U\left(m_{0}\right)$ where $U\left(m_{0}\right) \subset M$ is an open neighbourhood of $m_{0}$. As $M$ is compact there exists a finite covering $U\left(m_{1}\right), \ldots, U\left(m_{j}\right)$ of $M$ by such open neighbourhoods associated to $\tilde{a}^{\prime}$-invariant functions $f^{m_{1}}, \ldots, f^{m_{j}} \in C^{r}(P, \mathbf{R}) \cap L_{\tilde{v}^{\prime}}^{2}(P)$, and clearly $h=$ $\left(f^{m_{1}}\right)^{2}+\cdots+\left(f^{m_{j}}\right)^{2}$ is a solution.

We now resume the proof of Proposition 4.5.1. We first apply Lemma 4.5.2 to the $\widetilde{a}^{\prime}$-invariant function $h \in C^{r}(P, \mathbf{R}) \cap L_{\widetilde{v}^{\prime}}^{1}(P)$ yielded by Lemma 
4.5.4: there exists $\lambda \in C^{r}(G L(p)) \cap L^{1}(G L(p))$ such that in any trivialisation of $P$ we have $h_{m} \in \Pi(G L(p))(\lambda)$ for all $m \in M$. Let $G_{\lambda}=\left\{g_{0} \in\right.$ $G L(p) \mid \lambda\left(g_{0} g\right)=\lambda(g)$ for all $\left.g \in G L(p)\right\}$ be the stabilizer of $\lambda$ for $\Pi$. As $G L(p) / G_{\lambda}$ is a left $G L(p)$-space we can introduce the $G L(p) / G_{\lambda}$ fiber-bundle $P / G_{\lambda} \cong P \times_{G L(p)}\left(G L(p) / G_{\lambda}\right)$. An element $\bar{u}$ of $P / G_{\lambda}$ can be identified with a function on the fiber $P_{m}$ (by setting $f_{m}(g)=\lambda(g)$ ) where $m=\pi(u)$ ) which, in any trivialisation of the fiber $P_{m} \cong G L(p)$, belongs to $\Pi(G L(p)) \lambda$. We can thus assert:

The $\tilde{a}^{\prime}$-invariant function $\boldsymbol{h}$ on $\boldsymbol{P}$ defines pointwise an $\boldsymbol{a}^{\prime}$-invariant section $\sigma^{\prime}: M \rightarrow P / G_{\lambda}$. As $G_{\lambda}$ is compact, we will have proved the existence of an $a^{\prime}$-invariant $C^{r}$-Riemannian metric on $T \mathscr{F}$ provided that we can show that $\sigma^{\prime}$ is $C^{r}$ on $M$.

Fix $m_{0} \in M$. Let $\Phi: U\left(m_{0}\right) \times G L(p) \rightarrow \pi^{-1}\left(U\left(m_{0}\right)\right)$ be a smooth trivialization of $\pi^{-1}\left(U\left(m_{0}\right)\right)$ where $U\left(m_{0}\right)$ is an open neighbourhood of $m_{0}$ and let $\bar{\pi}: P / G_{\lambda} \rightarrow M$ denote the canonical projection of the fiber bundle $P / G_{\lambda}$. To the trivialization $\Phi$ corresponds the smooth quotient trivialization $\bar{\Phi}: U\left(m_{0}\right) \times G L(p) / G_{\lambda} \rightarrow \bar{\pi}^{-1}\left(U\left(m_{0}\right)\right)$ defined by $\bar{\Phi}(m, \bar{g})=\overline{\Phi(m, g)}$. We have just seen that for each $m \in U\left(m_{0}\right)$ there exists an $s(m) \in G L(p)$ such that

(1) $h(\Phi(m, g))=\lambda(s(m) g)$,

(2) $h \circ \Phi \in C^{r}\left(U\left(m_{0}\right) \times G L(p)\right)$,

(3) $(\Phi)^{-1} \circ \sigma^{\prime}(m)=(m, \overline{s(m)})$.

We now want to use those three items to prove that the function $\bar{s}: U\left(m_{0}\right) \rightarrow$ $G L(p) / G_{\lambda}$ defined by $\bar{s}(m)=\overline{s(m)}$ is $C^{r}$ on $U\left(m_{0}\right)$.

Lemma 4.5.5. $\bar{s}$ is continuous on $U\left(m_{0}\right)$.

Proof. Let $\left(m_{n}\right)_{n \in N}$ be a sequence of points of $U\left(m_{0}\right)$ which converges to $m_{0}$. We want to show that $\bar{s}\left(m_{n}\right) \underset{n \rightarrow \infty}{\longrightarrow} \bar{s}\left(m_{0}\right)$ (in $\left.G / G_{\lambda}\right)$. Assume that there exists a subsequence $\left(m_{n_{p}}\right)_{p \in N}$ such that $s\left(m_{n_{p}}\right) \underset{p \rightarrow \infty}{\longrightarrow} \infty$. Then on one hand

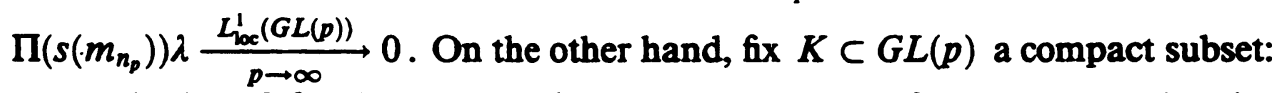
by continuity of $h \circ \Phi$, we have $h\left(\Phi\left(m_{n_{p}}, g\right)\right) \underset{p \rightarrow \infty}{\longrightarrow} f\left(\Phi\left(m_{0}, g\right)\right)$ pointwise and as $\left|h\left(\Phi\left(m_{n_{p}}, g\right)\right)\right| \leq C \in L^{1}(K)$ for all $p$ and all $g \in K$, by Lebesgue's

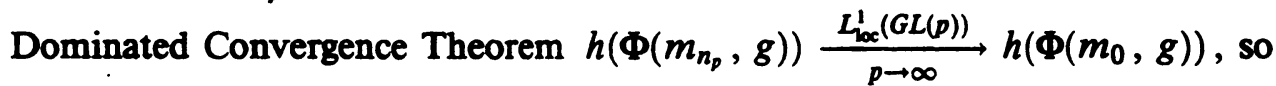
that $h\left(\Phi\left(m_{0},.\right)\right)=0$ which contradicts the assumption $h_{m_{0}} \neq 0$. We can therefore assume without loss of generality that the $s\left(m_{n}\right)$ lie in a compact set (so that the $\overline{s\left(m_{n}\right)}$ are also contained in a compact set). It is then enough to show that any convergent subsequence $\overline{s\left(m_{n_{p}}\right)}$ converges to $\overline{s\left(m_{0}\right)}$. Assume thus that $\overline{s\left(m_{n}\right)} \underset{n \rightarrow \infty}{\longrightarrow} \overline{g_{0}}$. Then there exist $g_{n} \in G L(p), g_{n} \underset{n \rightarrow \infty}{\longrightarrow} g$ such that $\overline{g_{n}}=\overline{s\left(m_{n}\right)}$ and $\bar{g}=\overline{g_{0}}$ (take a continuous section $j$ of the canonical projection $\bar{\pi}: G L(p) \rightarrow G L(p) / G_{\lambda}$ in a neighbourhood of $\overline{s\left(m_{0}\right)}$ and set

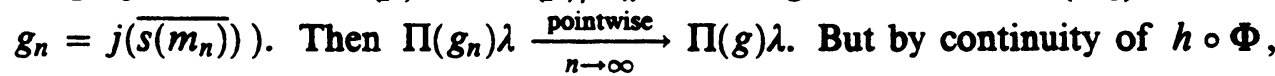
we also have $\Pi\left(g_{n}\right) \lambda=\Pi\left(s\left(m_{n}\right)\right) \lambda=h\left(\Phi\left(m_{n},.\right)\right) \underset{n \rightarrow \infty}{\stackrel{\text { pointwise }}{\longrightarrow}} h\left(\Phi\left(m_{n_{0}},.\right)\right)=$ $\Pi\left(s\left(m_{0}\right)\right) \lambda=\Pi(g) \lambda$, and thus $\bar{g}=\overline{s\left(m_{0}\right)}=\overline{g_{0}}$. 
It remains to show that $\bar{s}$ is $C^{r}$ on $U\left(m_{0}\right)$. This follows from the following lemma:

Lemma 4.5.6. Let $G$ be a connected Lie group, $\lambda \in C^{r}(G) \quad(1 \leq r \leq \infty)$ and $s: U \rightarrow G$ a map from an open subset $U \subset \mathbf{R}^{n}$ into $G$. Let $G_{\lambda}$ denote the closed Lie subgroup of $G$ defined by $G_{\lambda}=\left\{g_{0} \in G \mid \forall g \in G, \lambda\left(g_{0} g\right)=\lambda(g)\right\}$. Assume that

(1) the quotient map $\bar{s}: U \rightarrow G / G_{\lambda}$ given by $m \rightarrow \overline{s(m)}$ is continuous,

(2) the function $U \times G \rightarrow \mathbb{R}$ given by $(m, g) \rightarrow \lambda(s(m) g)$ is $C^{r}$.

Then the quotient map $\bar{s}: U \rightarrow G / G_{\lambda}$ given by $m \rightarrow \overline{s(m)}$ is also $C^{r}$.

Proof. $\lambda$ being constant on the right cosets of $G_{\lambda}$ we can define $\bar{\lambda}: G \backslash G_{\lambda} \rightarrow \mathbf{R}$ by $\bar{g} \rightarrow \bar{\lambda}(\bar{g})=\lambda(g)$. $\bar{\lambda}$ is also $C^{k}$ on $U$ (as we can look upon $G$ as a left $G_{\lambda}$-principal fiber bundle with base $G \backslash G_{\lambda}$ so that locally $\bar{\lambda}(\bar{g})=\lambda(\Phi(\bar{g}))$ where $\Phi: W \subset G \backslash G_{\lambda} \rightarrow G$ is smooth). Set $d=\operatorname{dim}\left(G \backslash G_{\lambda}\right)=\operatorname{dim}(G)-\operatorname{dim}\left(G_{\lambda}\right)$. Given $g_{1}, g_{2}, \ldots, g_{d}$ in $G$ we define a new $C^{r}$-function $\bar{\lambda}_{g_{1} g_{2} \ldots g_{d}}: G \backslash G_{\lambda} \rightarrow \mathbf{R}^{d}$ by

$$
\bar{\lambda}_{g_{1} g_{2} \ldots g_{d}}(\bar{g})=\left(\bar{\lambda}\left(\bar{g} g_{1}\right), \ldots, \bar{\lambda}\left(\bar{g} g_{d}\right)\right) \text {. }
$$

Sublemma. We can pick $g_{1}, g_{2}, \ldots, g_{d}$ in $G$ in such a way that $\bar{\lambda}_{g_{1} g_{2} \ldots g_{d}}$ is invertible in a neighbourhood of $\bar{e}$.

Proof of the sublemma. Let $g_{\lambda} \subset g$ be the Lie algebra of $G_{\lambda} \subset G$. We can assume without loss of generality that $\lambda$ is nonconstant, i.e., $G_{\lambda} \neq G$. Fix an inner product \langle\rangle on $\mathfrak{g}$, let \langle\rangle also denote the corresponding right-invariant metric on $G$, and introduce the function $V: G \rightarrow g$ defined by

$$
V(g)=R_{g}^{*}(\overrightarrow{g r a d} \lambda(g))
$$

(where $R_{g}$ denotes the right multiplication on $G$ and $\overrightarrow{g r a d}$ is considered with respect to the right-invariant metric (\rangle on $G$ ). We claim that

$$
V(g) \in g_{\lambda} \Longleftrightarrow \overrightarrow{\operatorname{grad}} \lambda(g)=0 \Longleftrightarrow V(g)=0 .
$$

Indeed assume that $V(g) \in g_{\lambda}$. Then $\operatorname{Exp}(t V(g)) \in G_{\lambda}$ for all $t \in \mathbf{R}$. Thus

$$
\begin{aligned}
0 & =\left.\frac{d}{d t}\right|_{t=0} \lambda(\operatorname{Exp}(t V(g)) \cdot g)=d \lambda(g)\left[\left.\frac{d}{d t}\right|_{t=0} \operatorname{Exp}(t V(g)) \cdot g\right] \\
& =\left\langle\overrightarrow{\operatorname{grad}} \lambda(g),\left(R_{g}\right)_{*}(V(g))\right\rangle_{T_{g} G}=\|\overrightarrow{\operatorname{grad}} \lambda(g)\|^{2} .
\end{aligned}
$$

We now show that there exist $g_{1}, g_{2}, \ldots, g_{d}$ in $G$ such that $V\left(g_{1}\right), \ldots$, $V\left(g_{d}\right)$ are linearly independent and satisfy $g=g_{\lambda} \oplus\left(V\left(g_{1}\right), \ldots, V\left(g_{d}\right)\right)$ where the second factor denotes the linear subspace spanned by $V\left(g_{1}\right), \ldots, V\left(g_{d}\right)$ : assume that $\{V(g) \in \mathfrak{g} \mid \overrightarrow{\mathrm{grad}} \lambda(g) \neq 0\}$ generates a subspace $\mathscr{H}=\left(V\left(g_{1}\right), \ldots\right.$, $\left.V\left(g_{k}\right)\right)$ with $k<d$. Then there exists a nontrivial $W \in\left(g_{\lambda} \oplus \mathscr{H}\right)^{\perp}=\mathscr{H}^{\perp} \cap \mathfrak{g}_{\lambda}^{\perp}$. Let $W$ denotes the corresponding right-invariant vector field on $G$ and fix $g \in G$. We have

$$
\begin{aligned}
\left.\frac{d}{d t}\right|_{t=t_{0}} \lambda(\operatorname{Exp}(t W) \cdot g) & =\left\langle\overrightarrow{\operatorname{grad}} \lambda\left(\operatorname{Exp}\left(t_{0} W\right) g\right), W\left(\operatorname{Exp}\left(t_{0}\right) g\right)\right\rangle_{T_{\left.\mathrm{Exp} t_{0} W\right) g} G} \\
& =\left\langle V\left(\operatorname{Exp}\left(t_{0} W\right) g\right), W\right\rangle_{g}=0
\end{aligned}
$$


(by right-invariance of the metric \langle\rangle and by definition of $W$ ). Since at $t=0$ we have $\lambda(\exp (0 W) g)=\lambda(g)$, for all $t \in \mathbb{R}$ we have $\operatorname{Exp}(t W) \in G_{\lambda}$ and thus $W \in g_{\lambda}$, which is a contradiction.

Now we see that $\bar{\lambda}_{g_{1} \ldots g_{d}}$ is invertible in a neighbourhood of $\bar{e}$. Let $k^{V\left(g_{i}\right)}$ be the killing vector field on $G \backslash G_{\lambda}$ associated to $V\left(g_{i}\right)$ and the canonical rightaction of $G$ on $G \backslash G_{\lambda}$. As $V\left(g_{1}\right), \ldots, V\left(g_{d}\right)$ is a basis of $g_{\lambda}^{\frac{1}{\lambda}} \subset g$ and $\left.d q\right|_{g_{\lambda}^{\frac{1}{2}}}$ : $g_{\lambda}^{\frac{1}{\lambda}} \rightarrow T_{e}\left(G \backslash G_{\lambda}\right)$ is a linear isomorphism, $\left(k^{V\left(g_{1}\right)}(\bar{e}), \ldots, k^{V\left(g_{d}\right)}(\bar{e})\right)$ is a basis of $T_{e}\left(G \backslash G_{\lambda}\right)$, and we have:

$$
\begin{aligned}
d\left(R_{g_{i}} \bar{\lambda}\right)\left(k^{V\left(g_{j}\right)}(\bar{e})\right) & =\left.\frac{d}{d t}\right|_{t=0} \bar{\lambda}\left(\bar{e} \cdot\left[\operatorname{Exp}\left(t V\left(g_{j}\right)\right)\right] g_{i}\right) \\
& =\left.\frac{d}{d t}\right|_{t=0} \lambda\left(\left[\operatorname{Exp}\left(t V\left(g_{j}\right)\right)\right] g_{i}\right) \\
& =\left\langle\overrightarrow{\operatorname{grad}} \lambda\left(g_{i}\right),\left[V\left(g_{j}\right)\right] \cdot g_{i}\right\rangle_{T_{s_{i}} G}=\left\langle V\left(g_{i}\right), V\left(g_{j}\right)\right\rangle_{g}
\end{aligned}
$$

(by right-invariance of the metric \langle\rangle$)$. As $\bar{\lambda}_{g_{1} \ldots g_{d}}=\left(R_{g_{1}} \bar{\lambda}, \ldots, R_{g_{d}} \bar{\lambda}\right)$, the matrix of $d \bar{\lambda}_{g_{1} \ldots g_{d}}(\bar{e})$ expressed on the basis $k^{V\left(g_{1}\right)}(\bar{e}), \ldots, k^{V\left(g_{d}\right)}(\bar{e})$ of $T_{e}\left(G \backslash G_{\lambda}\right)$ and the canonical basis of $\mathbf{R}^{d}$ is $\left(a_{i j}\right)=\left\langle V\left(g_{i}\right), V\left(g_{j}\right)\right\rangle_{g}$, which is invertible (as $V\left(g_{i}\right) \notin g_{\lambda}$ for each $i$ and $g=g_{\lambda} \oplus\left(V\left(g_{1}\right), \ldots, V\left(g_{d}\right)\right)$ ).

Proof of Lemma 4.5.6. Fix $m_{0} \in U$ and let $W(\bar{e}) \subset G \backslash G_{\lambda}$ be a neighbourhood of $\bar{e}$ on which $\bar{\lambda}_{g_{1} \ldots g_{d}}$ is invertible. As $G$ acts transitively on the left cosets of $G / G_{\lambda}$ there exists $g_{0} \in G$ such that $g_{0} \overline{s\left(m_{0}\right)}=\beta(\bar{e})$ where $\beta: G \backslash G_{\lambda} \rightarrow G / G_{\lambda}$ is the canonical diffeomorphism defined by $\beta\left(G_{\lambda} g\right)=g G_{\lambda}$. As $\bar{s}$ is continuous on $U$ there exists $U^{\prime}\left(m_{0}\right) \subset U$ such that $\beta^{-1}\left[g_{0} \bar{s}\left(U^{\prime}\left(m_{0}\right)\right)\right] \subset W(\bar{e}) \subset G \backslash G_{\lambda}$. By construction

$$
\bar{\lambda}_{g_{1} \ldots g_{d}}\left[\beta^{-1}\left(g_{0} \overline{s(m)}\right)\right]=\left(\lambda\left(g_{0} s(m) g_{1}\right), \ldots, \lambda\left(g_{0} s(m) g_{d}\right)\right)
$$

is a $C^{r}$-function of $U\left(m_{0}\right)$. By the Inverse Function Theorem we can invert $\bar{\lambda}_{g_{1} \ldots g_{d}}$ on $W(\bar{e})$ which implies that $m \mapsto \beta^{-1}\left(g_{0} \overline{s(m)}\right) \in C^{r}\left(U^{\prime}\left(m_{0}\right)\right)$ and thus that $m \mapsto \overline{s(m)} \in C^{r}\left(U\left(m_{0}\right)\right)$.

\section{REFERENCES}

1. Robert J. Zimmer, Ergodic theory and semisimple groups, Monographs Math., vol. 81, Birkhäuser, Boston, 1984.

2. Lattices in semisimple groups and invariant geometric structures on compact manifolds, Discrete Groups in Geometry and Analysis, Papers in Honor of G.D. Mostow on His Sixtieth Birthday, Prog. Math., vol. 67, Birkhăuser, Boston, 1987, pp. 152-210.

3. Engodic theory and the automorphism group of a G-structure, Group Representations, Ergodic Theory and Mathematical Physics (C. C. Moore, ed.), Springer-Verlag, Berlin and New York, 1987, pp. 247-278.

4. - On the algebraic hull of an automorphism group of a principal bundle, Comment Math. Helv. 65 (1990), 375-387.

5. Pierre de la Harpe et Alain Valette, La propriete $(T)$ de Kazhdan pour les groupes localement compacts, 175, Astérisque, Soc. Math. France, 1989.

6. A. Katok and J. Lewis, Local rigidity for certain groups of toral automorphisms, Preprint.

Universite de Metz, D. M. I., Ile du Saulcy, 57045 Metz Cedex 01, France

E-mail address: seydouxedmi.univ-metz.Ir 